\title{
THE TISSUE RENIN-ANGIOTENSIN SYSTEM (tRAS) AND THE IMPACT OF ITS INHIBITION ON INFLAMMATION AND BONE LOSS IN THE PERIODONTAL TISSUE
}

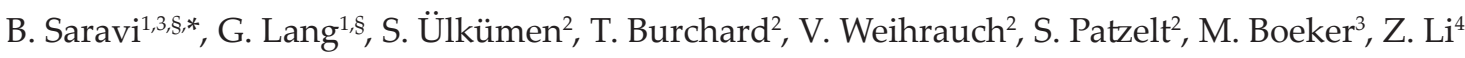 \\ and J. P. Woelber ${ }^{5}$ \\ ${ }^{1}$ Department of Orthopaedics and Trauma Surgery, Faculty of Medicine, \\ Albert Ludwig University of Freiburg, Germany \\ ${ }^{2}$ Department of Prosthetic Dentistry, Centre for Dental Medicine, \\ University Medical Centre Freiburg, Freiburg, Germany \\ ${ }^{3}$ Institute of Medical Biometry and Statistics, Faculty of Medicine and \\ University Medical Centre Freiburg, Freiburg, Germany \\ ${ }^{4} \mathrm{AO}$ Research Institute Davos, Davos, Switzerland \\ ${ }^{5}$ Department of Operative Dentistry and Periodontology, Medical Centre, \\ University of Freiburg Faculty of Medicine, Freiburg, Germany \\ $\S$ These authors contributed equally
}

\begin{abstract}
Recently, the existence of the tissue renin-angiotensin system (tRAS) has been described for multiple tissues in humans, suggesting its fundamental role in the progression of inflammation and fibrosis. Evidence arises that tRAS might have an impact on the progression of periodontitis and bone loss. However, neither the role of tRAS nor its impact as a therapeutic target have been systematically evaluated for periodontal tissue. The present study sought to characterise tRAS in the periodontal tissue and the effect of its inhibition on periodontal inflammation and bone loss. This systematic review was performed according to the preferred reporting items for systematic reviews and meta analyses (PRISMA) statement. Literature was searched using Web of Science core collection (Web of Science), Medline (Ovid), Cochrane central register of controlled trials (Ovid), Cochrane database of systematic reviews (Ovid), Google Scholar databases and the references of the retrieved studies in March 2020. Information on study design, sample size, population, procedure, type of intervention, observation time, as well as information on sources of bias, was extracted and evaluated. From 455 identified articles, 17 were included in the qualitative synthesis and 11 were included in the quantitative synthesis. Outcomes of studies indicated that the inhibition of tRAS components led to a reduction of periodontal bone loss and inflammation, dependent on the inhibitor used. The findings suggested an important role of tRAS in the periodontal tissue and indicate a potential therapeutic approach for periodontal diseases.
\end{abstract}

Keywords: Periodontal bone loss, periodontitis, inflammation, renin-angiotensin system, degeneration, regeneration, osteogenesis.

*Address for correspondence: Dr Babak Saravi, Department of Orthopaedics and Trauma Surgery, Medical Centre, Faculty of Medicine, Albert Ludwig University of Freiburg, Germany.

Telephone number: + 49761 270-27830 Fax number: + 49761 270-27830 Email: babak.saravi@jupiter.unifreiburg.de

Copyright policy: This article is distributed in accordance with Creative Commons Attribution Licence (http://creativecommons.org/licenses/by-sa/4.0/).

\section{Introduction}

Severe periodontitis is one of the most prevalent human diseases and the major cause of tooth loss in adults worldwide, with an overall prevalence of approximately $11 \%$ (Dye, 2012; Frencken et al., 2017; Kassebaum et al., 2014; Petersen and Ogawa, 2012). The global cost of lost productivity from periodontitis has been estimated to be 54 billion \$US annually (Marcenes et al., 2013; Tonetti et al., 2017). The disease is characterised by progressive inflammation, loss of periodontal ligament, and alveolar bone loss (Pihlstrom et al., 2005). The infection of the periodontal tissue is known as the main aetiological factor of this inflammation process (Darveau, 2010). However, the dysregulated cellular 
pathways promoting the inflammatory vicious circle are host mediated (Darveau, 2010; Kantarci et al., 2006). Current treatment strategies include pharmacological approaches, such as systemic and local antibiotic therapy and antiseptic therapy, mechanical anti-infective therapy, and surgical interventions (Krayer et al., 2010). These approaches focus on the primary aetiology of periodontal disease: bacterial infection. Another therapeutical approach is the host modulation therapy (HMT), based on the management of periodontal disease through the control of host immune and inflammatory response (Greenwell, 2001; Krayer et al., 2010; Reddy et al., 2003). Non-steroidal anti-inflammatory drugs (NSAIDs), anti-cytokine, and biological therapies and inhibition of matrix metalloproteinase (MMP) activity are some described host modulation therapeutics for periodontal diseases (Preshaw, 2018). Furthermore, diet is an important factor in host modulation and progression of periodontal diseases (Woelber and Tennert, 2020).

Taking account of the socioeconomic relevance of periodontal diseases, new biomolecular therapies targeting the inflammatory and destructive processes are crucial when other less invasive therapeutic approaches fail. As inflammation induces and triggers these degenerative pathways, profound knowledge on the proinflammatory interactions helps to find optimally targeted therapeutics and control critical host response reactions.

Nearly a hundred years after the first description of the renin-angiotensin system (RAS) by Tigerstedt and Bergmann, the concept of a local or tissue reninangiotensin system (tRAS) emerged in the scientific world (Dzau and Re, 1994; Lind paintner and Ganten, 1991; Paul et al., 1992; Tigerstedt and Bergman, 1898). Hence, an intracellular-renin-angiotensin system (iRAS) and an intercellular-renin-angiotensin system (intRAS) were introduced interacting with the classic circulating humoral RAS (Abadir et al., 2011; Alzayadneh and Chappell, 2015; Filipeanu et al., 2001; Gwathmey et al., 2012). Angiotensin II (ATII), as the most important effector of the tRAS, is involved in multiple intracellular pathways including mitochondrial and nuclear signalling (Filipeanu et al., 2001; Re, 2018). Furthermore, the iRAS - as the regulatory arm of the tRAS - determines in which way the cell will react, dependent on extracellular signals and the intracellular ATII concentration (Filipeanu et al., 2001). Moreover, the angiotensin II type 1 receptor (AT1R) has been described in multiple intracellular compartments - such as mitochondria, nucleus and lysosomes - and is responsible for many intracellular processes, such as aging, cell proliferation, and increase of mRNA expression (Valenzuela et al., 2016; Villar-Cheda et al., 2017). Further, anti-proliferative and anti-inflammatory, or proliferative and pro-inflammatory, cell responses are reported to be directly related to the cellular ATII concentration (Filipeanu et al., 2001; Villar-Cheda et al., 2017). Additionally, studies indicated a central trend of the tRAS signalling: the dominance of the AT1R mediated pathway leads to inflammation and oxidative stress, while the intracellular AT1R signalling processes and the angiotensin II type 2 receptor (AT2R) pathway seem to have modulating roles (Chabrashvili et al., 2003; Valenzuela et al., 2016; Villar-Cheda et al., 2017). The existence and importance of a tRAS have been described for multiple human tissues such as the brain, heart, liver, pancreas, musculoskeletal and adipose tissue (Karlsson et al., 1998; van Kats et al., 1998; Lu et al., 2007; Moulik et al., 2002; Zhao et al., 2019) (also congress abstract: Lang et al., 2018. The tissue-renin-angiotensinsystem of the human intervertebral disc. Deutscher Kongress für Orthopädie und Unfallchirurgie. DOI: 10.3205/18DKOU478).

Recent studies implicate a fundamental role of the tRAS in metabolic diseases and host-mediated inflammation reactions. For example, a link between the tRAS and diabetes, obesity, atherosclerosis, Alzheimer's disease, kidney, vascular and heart diseases have been described (Biancardi et al., 2017; Gebre et al., 2018; Montecucco et al., 2009; Ramalingam et al., 2017; Remuzzi et al., 2005; Ruiz-Ortega et al., 2001). The complex interactions of tRAS with the circulatory renin-angiotensin system could be one explanation for the association between periodontitis and cardiovascular diseases (Martin-Cabezas et al., 2016; Santos et al., 2015; Viafara-Garcia et al., 2019).

The presence of a tRAS has also been recently described for bone and connective tissues (Gebru et al., 2013; Morimoto et al., 2013) (also congress abstract: Lang et al., 2018. See earlier paragraph). Additionally, the existence of a tRAS in the periodontal and gingival tissue has been described by numerous studies (Berggreen and Heyeraas, 2003; Ohuchi et al., 2002; Ohuchi et al., 2004; Santos et al., 2009). It is suggested that this system is a key player in bone remodelling and modulation of host immunoreactions (Gebru et al., 2013). Fig. 1 illustrates potential tRAS mechanisms in the presence of periodontitis-associated bacteria. It is well known that stimulation of toll-like receptors (TLRs) in periodontal fibroblasts, through pathogens, leads to an activation of the transcription factor nuclear factor-kappa B (NF-kB), an increase of reactive oxygen species (ROS) and the expression of pro-inflammatory molecules, such as interleukin (IL)-1ß (Gabriele et al., 2017; Miggin, 2006; Nadlonek et al., 2013; Wu, 2006). A recent study showed crosstalks between TLRs and ATII with an upregulation of TLRs and an increase of NF-kB, monocyte chemoattractant protein 1 (MCP1), and IL-6, all are important factors of the host-mediated immuneresponse (Lv et al., 2015). The induction of adhesion molecules and proinflammatory mediators, such as tumour necrosis factor-alpha (TNF- $\alpha$ ) and IL$1 \beta$, promotes the host-mediated process, leading to bone resorption and tissue destruction (Graves and Cochran, 2003). Shimizu et al. showed that ATII induces the expression of NF-kB ligand (RANKL) 

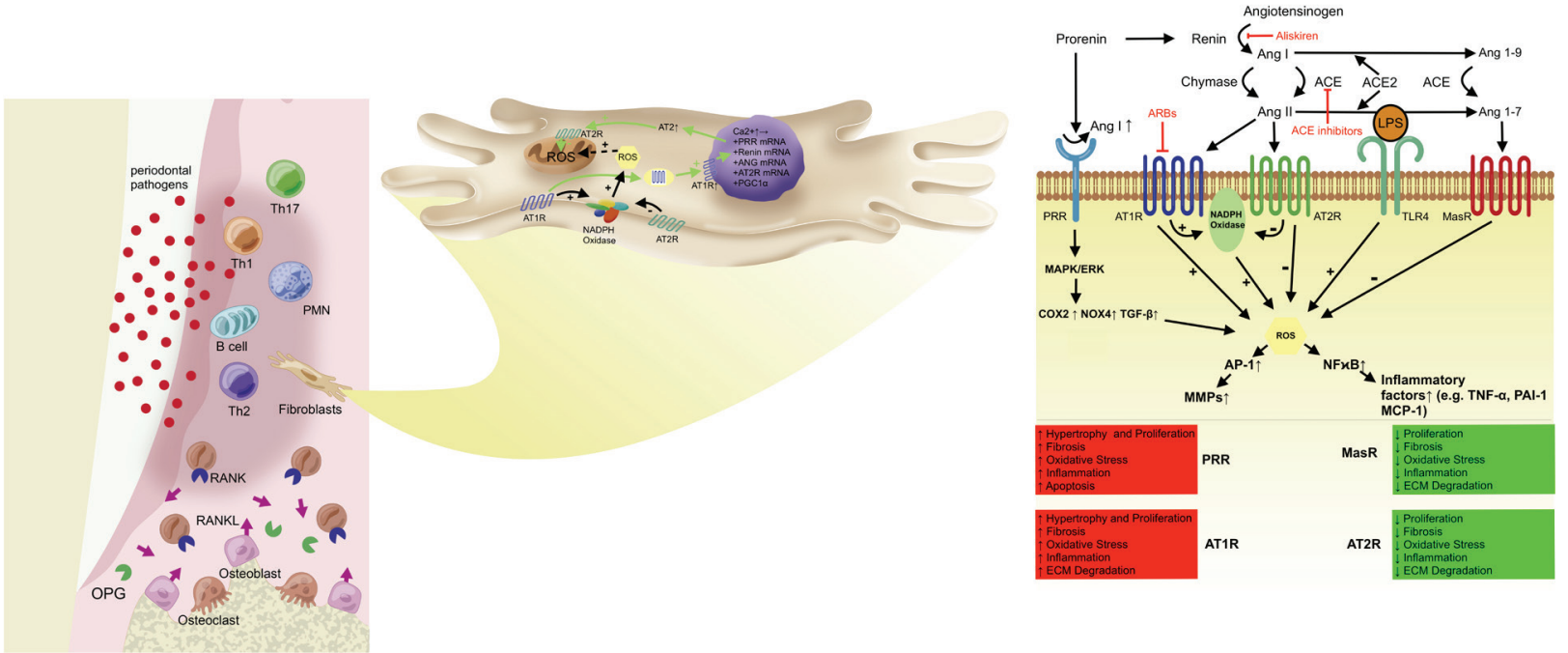

Fig. 1. Illustration of possible tRAS mechanisms modulating the host response in periodontal tissue after periodontal pathogen invasion. Pathogen invasion leads to a host-mediated cascade of inflammatory response involving an increase of AT1R activity, production of ROS, upregulation of NF-kB, and secretion of inflammatory- and tissue degrading molecules. Proinflammatory molecules lead to an upregulation of RANKL and an increase of the RANKL/OPG ratio, promoting periodontal bone loss. This upregulation of the proinflammatory pathway by the prorenin receptor (PRR), AT1R and TLR4 (stimulated by LPS of periodontal pathogens) is controlled by the AT2R, mas receptor (MasR) and intracellular RAS signalling (Nakamura et al., 2011; Villar-Cheda et al., 2017; Zhao et al., 2019).

in osteoblasts, leading to activation of osteoclasts, whereas the effect was blocked by an AT1R inhibitor (Olmesartan) (Shimizu et al., 2008). Furthermore, ATII has mitogenic properties in periodontal cells and that stimulation could lead to cell proliferation, generation of ROS, an increase of NF-kB and subsequent stimulation of various proinflammatory molecules, such as Prostaglandin E2 and IL-1 $\beta$, promoting the periodontal inflammation (Gabriele et al., 2017; Lundergan et al., 1999; Nakamura et al., 2011; Segawa et al., 2003). A knockdown of the AT1R in periodontal fibroblasts impaired the secretion of IL-1 $\beta$, IL-6 and IL-8, all important mediators of periodontal inflammation (Gabriele et al., 2017). Finally, studies revealed that inhibition of tRAS pathways lead to an increase in bone mass and a reduction of fracture risks (Lynn et al., 2006; Nakagami and Morishita, 2009; Shimizu et al., 2008; Solomon et al., 2011; Zhang et al., 2014). These observations indicate that a tRAS exists in the periodontal tissue and might be an important target approach.

Based on these findings, this systematic review aimed to answer the following question: What is the impact of the tRAS inhibition on inflammation and bone loss in the periodontal tissue?

\section{Materials and Methods}

\section{Review protocol}

This review was conducted based on the preferred reporting items for systematic reviews and metaanalyses (PRISMA) Statement and our systematic review protocol was specified using SYRCLE's systematic review protocol for animal intervention studies (Tricco et al., 2018; de Vries et al., 2015). The review protocol was registered in PROSPERO (international prospective register of systematic reviews) hosted by the UK's National Institute for Health Research (NHS), University of York, Centre for Reviews and Dissemination, under the code CRD42020178423.

\section{Search strategy}

Literature was searched up to March 2020, focusing on animal studies, tRAS and its inhibitors [angiotensin II type 1 receptor inhibitors (ARBs)], angiotensinconverting-enzyme inhibitors (ACE-inhibitors), renin inhibitors, periodontitis, and alveolar bone loss. Initially, a combined medical-subject headings $(\mathrm{MeSH})$ and free-text term electronic search of the literature was performed using Medline (Ovid), Cochrane central register of controlled trials (CENTRAL, Trials) (Ovid), and Cochrane database of systematic reviews (CDSR) (Ovid). Subsequently, the search strategy used in Medline was translated into an appropriate format for searching the Web of Science core collection (Web of Science) with a freetext term search. Text-word truncation was applied to retrieve all forms of the search terms and Boolean logical operators were used to combine the search results (Table 1). Furthermore, a supplementary free-text term search was performed using Google Scholar as well as hand searches of the references of the selected studies. All studies published before March $25^{\text {th }}, 2020$ were considered. No language restrictions were applied and retrieved articles in foreign languages were translated. 


\section{Eligibility criteria}

The following inclusion criteria were adopted, based on the PICOS process: (P) Population where animals in which experimental periodontitis or similar methods were used to investigate the desired tRAS inhibition outcomes. The intervention (I) was an inhibition of tRAS using ARBs, ACE-inhibitors, or renin inhibitors. The comparator (C) had no tRAS inhibition. The primary outcome $(\mathrm{O})$ was (histo-) morphometric measurements of bone loss and bone volume. The secondary outcomes were the number of osteoclasts and immunoinflammatory/oxidative stress markers (e.g. CRP, TNF- $\alpha, \mathrm{MPO})$ and bone remodelling markers (e.g. RANKL, OPG). Study designs (S), with an experimental examination of tRAS inhibition in the periodontal tissue in animals, were included without restrictions to retrieve all available evidence.

The following exclusion criteria were applied:

1. human studies and in vitro studies

2. review articles, case studies, method-comparison studies

3. Comparator and intervention group are not similar, e.g. One of the groups has study characteristics which could affect $t$ RAS outcomes (e.g. hypertension or other cardiovascular diseases))

4. studies which focussed on the examination of adjacent structures (e.g. tooth) or related diseases (e.g. pulpitis) there was no matched control group.

To do justice to the nature of preclinical animal studies (e.g. often multiple experiments in one animal study, multiple intervention arms) and to retrieve the maximum information from the current evidence, the desired data corresponding to a PICOS research question was extracted - instead of excluding the studies relating to multiple intervention groups or experimental settings, regardless of the fact that groups or experimental settings that are part of the study would not meet our inclusion criteria. In the studies reporting data from multiple groups with multiple interventions, baseline characteristics, or experimental setups, only the intervention group was considered and its matched comparator that met our inclusion and exclusion criteria.

\section{Study selection and data extraction}

The selection of studies was performed in two-steps. Firstly, two reviewers (B.S. and S.U.) independently screened the titles and abstracts for eligibility. Subsequently, an independent full-text analysis was performed by the two reviewers. The reasons for exclusion were recorded in the second step. Any disagreement between reviewers was solved by consensus with a third reviewer (T.B.). Agreement between reviewers was assessed using $\kappa$ statistics (Cohen, 1960).

Data extraction was performed by the two independent reviewers using a data extraction form which contained the following data extraction parameters:

- article and study identifiers: author, country, year of publication, objective

- setting and population: sample size of comparator and intervention groups, animal species, weight,

Table 1. Search strategy. exp: explode function; TS: Topic; NEAR: proximity operator; OR: boolean operator; AND: boolean operator; *: truncation symbol; .mp: multi-purpose field search.

\begin{tabular}{|c|c|c|c|}
\hline $\begin{array}{c}\text { Search } \\
\text { date }\end{array}$ & Database & Search strategy & Results \\
\hline $25 / 03 / 2020$ & $\begin{array}{l}\text { Ovid Medline }{ }^{\circledast} \text { and Epub } \\
\text { ahead of print, In-process \& } \\
\text { other non-indexed citations, } \\
\text { Daily and versions }{ }^{\circledast}<1946 \text { to } \\
\text { present> } \\
\text { EBM reviews - Cochrane } \\
\text { central register of controlled } \\
\text { trials (CENTRAL, Trials) via } \\
\text { Ovid <inception to present> } \\
\text { EBM reviews - Cochrane } \\
\text { database of systematic } \\
\text { reviews (CDSR) via OVID } \\
\text { <inception to present> }\end{array}$ & $\begin{array}{l}\text { 1. exp periodontitis/ } \\
\text { 2. exp periodontal diseases/ } \\
\text { 3. exp gingiva/ } \\
\text { 4. exp periodontium/ } \\
\text { 5. exp alveolar bone loss/ } \\
\text { 6. periodont*.mp } \\
\text { 7. exp alveolar process/ } \\
\text { 8. marginal bone loss*.mp } \\
\text { 9. alveolar bone loss*.mp } \\
\text { 10. exp oral health/ } \\
\text { 11. mandib*.mp } \\
\text { 12. maxill*.mp } \\
\text { 13. attachment loss*.mp } \\
\text { 14. exp Tooth/ } \\
\text { 15. or/1-14 } \\
\text { 16. exp angiotensin-converting enzyme inhibitors/ } \\
\text { 17. exp angiotensin II/ } \\
\text { 18. exp angiotensin II type } 1 \text { receptor blockers/ } \\
\text { 19. exp angiotensin receptor antagonists/ } \\
\text { 20. angiotensin*.mp. } \\
\text { 21. ARBs.mp } \\
\text { 22. ACE.mp } \\
\text { 23. RAAS.mp } \\
\text { 24. or/16-23 } \\
\text { 25. } 15 \text { and } 24 \\
\end{array}$ & $\begin{array}{r}33.041 \\
92.750 \\
18.515 \\
46.601 \\
11.326 \\
105.613 \\
14.406 \\
2.108 \\
12.516 \\
16.571 \\
123.883 \\
121.926 \\
6.748 \\
91.554 \\
398.501 \\
49.491 \\
37.797 \\
19.915 \\
26.137 \\
145.504 \\
4.197 \\
40.267 \\
3.227 \\
168.047 \\
178 \\
\end{array}$ \\
\hline $25 / 03 / 2020$ & $\begin{array}{l}\text { Web of Science core } \\
\text { collection (Web of Science) }\end{array}$ & $\begin{array}{l}\text { \#1 TS = ((periodontitis) OR (gingiva) OR (periodontal NEAR/5 disease*) OR (periodontium) } \\
\text { OR (periodont*) OR (oral NEAR/5 health) OR (tooth) OR ((bone* OR osteo* OR alveolar) } \\
\text { NEAR/5 (alveolar OR maxill* OR mandib* OR jaw OR bucc* OR marginal OR process OR } \\
\text { ridge OR attachment) NEAR/5 (loss OR resorption OR defect* OR density OR atroph* OR } \\
\text { lyses OR osteolys* OR volume)) } \\
\text { \#2 TS = ((angiotensin-converting enzyme inhibitors) OR (angiotensin II) OR (angiotensin II } \\
\text { type } 1 \text { receptor blockers) OR (angiotensin receptor antagonists) OR (ARBs) OR (*sartan) OR } \\
\left.\text { (ACE) OR (RAAS) OR (angiotens }{ }^{*}\right) \text { OR ((angiotensin* OR AT1 OR AT-1) NEAR/5 (receptor* } \\
\text { OR antagonist* OR type* OR convert* OR inhibit* OR blocker*))) } \\
\text { \#3 \#1 AND \#2 }\end{array}$ & 183.501 \\
\hline $25 / 03 / 2020$ & $\begin{array}{l}\text { Google Scholar and hand } \\
\text { searching of retrieved studies }\end{array}$ & & 16 \\
\hline
\end{tabular}


age, sex, and experimental setting to examine tRAS inhibition, observation period, ethic statement (yes/no)

- intervention: name, dose, type of tRAS inhibition and duration of the inhibition

- method: method of outcome measurement

- outcome and results: reported outcomes (intervention $v s$. comparator)

- author's conclusion

- reviewer comments.

The extraction of data to assess the risk of bias was performed separately and is shown in the risk of bias section.

\section{Synthesis of results}

Methods for direct treatment comparisons to assess secondary outcomes

Initially, a pairwise meta-analysis was performed to assess study outcomes quantitatively for every outcome with at least three reporting studies. Pairwise meta-analysis was conducted using Review Manager (RevMan, version 5.3, Copenhagen: The Nordic Cochrane Centre, The Cochrane Collaboration, 2011). Because of the heterogeneity of the included studies, especially the different duration of tRAS inhibition, different doses of inhibitors, different inhibitors, different species, and different methodological settings, it was decided to use a random-effects model meta-analysis (Higgins J, Green S (editors) (2011) Cochrane handbook for systematic reviews of interventions Version 5.1.0 [updated March 2011]; The Cochrane Collaboration (2003) Review Manager (RevMan) version 4.2.10 [computer program]. The Nordic Cochrane Centre, The Cochrane Collaboration; 2003; The Cochrane Collaboration, 2011: Available from http://handbook. cochrane.org.). Hedge's $g$ was used to calculate the standardised mean difference (SMD). Confidence intervals (CI) were used to measure the degree of unncertainty or certainty using a confidence level of $95 \%$ (95\% CI). To evaluate the heterogeneity of the included studies $I^{2}$ statistics were used. Values of $I^{2}$ more than $25 \%, 50 \%$ and $75 \%$ were specified as low, moderate and high heterogeneity, respectively (Higgins et al., 2003). No subgroup or sensitivity analysis was performed due to the limited number of animal studies, but a qualitative interpretation of possible heterogeneity sources was provided if the heterogeneity was judged high. Cohen's $D$ was used to evaluate the effect size, and a $D$ between 0.2 and 0.5 was specified as a small effect, a $D$ between 0.5 and 0.8 as a medium effect and a $D$ greater than 0.8 as a large effect. To visualise differences between intervention arm and comparator for secondary outcomes with regards to the tRAS inhibitor class and the duration of intervention, values were calculated for the secondary outcomes as a percentage of the reference value (untreated control group) and a heatmap was produced using GraphPadPrism version 8.4.2 (GraphPad Software, LLC.).
Methods for mixed and network comparisons of multiple intervention arms for the primary outcome

Network geometries were presented as spiderlike web-charts, to show the connections between the different pharmacological intervention arms regarding the periodontal bone loss. A network meta-analysis was conducted for all treatment arms, including the different doses and subgroups of the tRAS inhibitors, as well as network meta-analysis of pooled tRAS inhibitor treatments (Control vs. ARB vs. ACE-inhibitor vs. renin-inhibitor). Because of the heterogeneity of animal studies and the comparison of multiple treatment arms, the approach based on random-effects multivariate meta-regression "mvmeta" was used as presented by White et al. that applies the frequentist method for estimation in the network meta-analysis (White et al., 2012). The network analysis was performed using Stata Statistical Software Release 15 (StataCorp. 2011, College Station, TX, USA). Furthermore, the network package, the "mvmeta" command and self-programmed Stata routines (Web ref. 1) were used (Chaimani et al., 2013; White, 2015). To assess the relative treatment rankings, the "surface under the cumulative ranking" (SUCRA) curve was used as well as mean ranks (Salanti et al., 2011). From the three assumptions: similarity, transitivity and consistency, to be satisfied a priori for the network meta-analysis similarity was satisfied by the PICOS procedure, consistency by statistical methods using global ("design-by treatment approach") and local approaches ("loop-specific approach") to assess inconsistency, and transitivity by logical interpretation of outcome interferences and the statistical consistency tests (Cipriani et al., 2013).

\section{Data management and assessment of treatment effects}

If studies contained multiple experimental groups with the same tRAS inhibitor compared to a single control, the experimental groups were combined to create a single pair-wise comparison to avoid unit-of-analysis error due to double-counts in the shared-control groups. If studies contained multiple experimental groups, with different tRAS inhibitors compared to a single control group, the control group was split (Higgins J, Green S (editors) (2011) Cochrane handbook for systematic reviews of interventions Version 5.1.0). If studies contained multiple experimental groups, with matched control groups, the experimental groups were considered as separate experiments. Multiple animal subgroups within a single study (e.g. different species or strains) were included as independent experimental groups with independent SMDs. Because the focused outcomes were presented as continuous data and measures of outcomes were presented in a variety of ways (i.e. outcomes measured using different scales and methods) the SMD was used as an effect measure. When the included studies only reported the standard error of the mean, the standard deviation 
was calculated first (Altman and Bland, 2005). When only bone volume measurements were shown in the manuscripts, the bone loss was calculated from the baseline value of untreated study groups, where applicable. Where necessary, means and standard deviations (or standard errors of the mean) were extracted from figures of the included manuscripts using WebPlotDigitizer (Web ref. 2).

\section{Assessment of the risk of bias}

SYRCLE's Risk of Bias tool was used to assess the risk of bias for all included studies (Hooijmans et al., 2014). This tool was adapted from the Cochrane risk of bias tool for randomised controlled trials with human participants (Higgins J, Green S (editors) (2011) Cochrane handbook for systematic reviews of interventions Version 5.1.0). The following ten methodological domains were examined:

1. Selection bias:

a) Was the allocation sequence adequately generated and applied?"

b) Were the groups similar at baseline or were they adjusted for confounders in the analysis? c) Was the allocation adequately concealed?

2. Performance bias:

a) Were the animals randomly housed during the experiment?"

b) Were the caregivers and/or investigators blinded from knowledge which intervention each animal received during the experiment?

3. Detection bias:

a) Were animals selected at random for outcome assessment?

b) Was the outcome assessor-blinded?

4. Attrition bias: Were incomplete outcome data adequately addressed?

5. Reporting bias: Are reports of the study free of selective outcome reporting?

6. Other sources of bias: Was the study free of other problems that could result in high risk of bias?

The items in the RoB tool were scored with "high" (high risk of bias), "low" (low risk of bias), and "unclear" (the item was not reported and the risk of bias could not be examined). Two independent investigators (B.S. and S.U.) performed a quality assessment of all included studies. Disagreements were resolved by discussion with a third reviewer (T.B.).

\section{Results}

\section{Study selection and study characteristics}

A total of 455 articles were identified and assessed for eligibility. After removing duplicates and screening abstracts and titles, 423 studies were excluded. In the following list, 15 studies were excluded based on the full-text analysis. Overall, 17 studies (Araujo et al., 2013a; Araujo et al., 2013b; Araújo et al., 2014; Dionisio et al., 2019; Goncalves-Zillo et al., 2013; Li et al., 2019; Matos et al., 2013; Matos et al., 2014; Matos et al., 2015; Matos et al., 2016; Matos et al., 2019; Moura et al., 2016; Mulinari-Santos et al., 2019; Oliveira et al., 2019; Queiroz-Junior et al., 2015; Santos et al., 2015; Suda et al., 2013) were included in the qualitative synthesis (Fig. 2). Of these, 11 studies reported the primary outcome with extractable quantitative data and were included in the network meta-analysis (Araujo et al., 2013a; Araujo et al., 2013b; Araújo et al., 2014; Dionisio et al., 2019; Goncalves-Zillo et al., 2013; Li et al., 2019; Mulinari-Santos et al., 2019; Oliveira et al., 2019; Queiroz-Junior et al., 2015; Santos et al., 2015; Suda et al., 2013). In the secondary outcome, quantitative synthesis was assessed - based on at least three reports - and included studies with extractable quantitative data: malondialdehyde (MDA), myeloperoxidase (MPO), glutathione (GSH), IL-1 $\beta$, IL-10, TNF- $\alpha$, RANKL and number of tartrateresistant acid phosphatase positive (TRAP+) cells/ osteoclasts. Based on these data, 11 studies were included in the pair-wise meta-analysis (Araujo et al., 2013b; Araújo et al., 2014; Li et al., 2019; Matos et al., 2013; Matos et al., 2014; Matos et al., 2015; Moura et al., 2016; Oliveira et al., 2019; Queiroz-Junior et al., 2015; Suda et al., 2013). Two studies did not report on the sample sizes (Matos et al., 2016; Matos et al., 2019). No answer was received on contacting the authors, thus these two studies were only included in the qualitative synthesis. The resulted value of $\kappa$ statistic test to evaluate the agreement between the reviewers was 0.92 , indicating an excellent agreement.

\section{Baseline characteristics}

All studies included in this systematic review were published after 2013, each reporting data from between 10 and 40 animals. Data from an overall sample of 390 animals were assessed, but noting also that two of the studies did not report on sample sizes. 6 animal model studies reported data from mice and 12 from rats. One study reported data from both mice and rats. The baseline characteristics of the included studies are shown in Table 2.

\section{Qualitative synthesis of study characteristics}

Seven studies used the application of a ligature to induce periodontitis (ligature induced experimental periodontitis), 2 studies used an infection with Porphyromonas gingivalis, 1 study used an infection with Aggregatibacter actinomycetemcomitans, 5 studies used lipopolysaccharide (LPS) application, 1 study compared physiological conditions and 1 study used orthodontic force application as the examination method (Table 3). The ligature induced periodontitis model was based on the placement of a sterile nylon thread or silk ligature around the submarginal position of the maxillary or mandibular molars. For the bacteria-induced periodontitis model with Porphyromonas gingivalis, the strain was grown under anaerobic conditions at $37^{\circ} \mathrm{C}$. Li et al. then added sterile $2 \%(\mathrm{w} / \mathrm{v})$ carboxymethylcellulose to the bacteria, mixed the suspension, and administered 


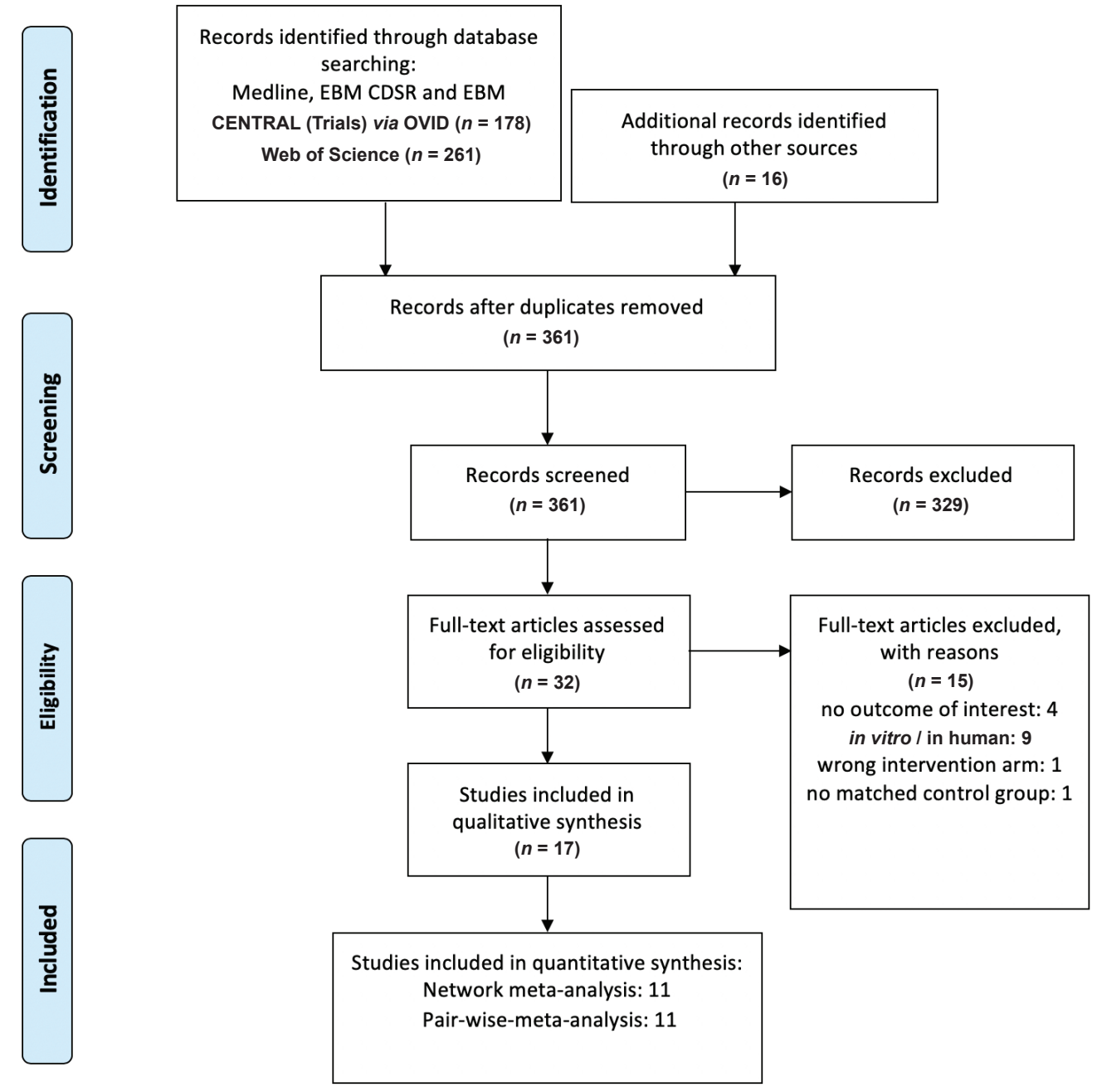

Fig. 2. PRISMA flow diagram of the selection process.

Table 2. Baseline characteristics of the included studies.

\begin{tabular}{|c|c|c|c|c|c|c|c|}
\hline \multicolumn{8}{|c|}{ Animal model: Rat } \\
\hline Reference & Country & Strain/animal & Sex & Age & Weight & $\begin{array}{l}\text { Total number: } \\
\text { control and } \\
\text { intervention }\end{array}$ & $\begin{array}{c}\text { Ethics } \\
\text { committee }\end{array}$ \\
\hline Araújo et al., 2013 & BRA & Wistar & $\mathrm{M}$ & NA & $180-220 \mathrm{~g}$ & 40 & Yes \\
\hline Araújo et al., 2013 & BRA & Wistar & M & NA & $180-220 \mathrm{~g}$ & 40 & Yes \\
\hline Araújo et al., 2014 & BRA & Wistar & $\mathrm{M}$ & NA & $180-220 \mathrm{~g}$ & 40 & Yes \\
\hline Dionísio et al., 2019 & BRA & Wistar & M & $60-90 \mathrm{~d}$ & $320-400 \mathrm{~g}$ & 15 & Yes \\
\hline Gonçalves-Zillo et al., 2013 & BRA & Wistar & $\mathrm{M}$ & 12 weeks & NA & 21 & Yes \\
\hline Matos et al., 2013 & VEN & Sprague-Dawley & $\mathrm{M}$ & NA & $280-300 \mathrm{~g}$ & 10 & Yes \\
\hline Matos et al., 2014 & VEN & SpragueDawley & NA & NA & $280-300 \mathrm{~g}$ & 27 & Yes \\
\hline Matos et al., 2015 & VEN & SpragueDawley & $\mathrm{M}$ & NA & $280-300 \mathrm{~g}$ & 27 & Yes \\
\hline Matos et al., 2016 & VEN & SpragueDawley & $\mathrm{M}$ & NA & $280-300 \mathrm{~g}$ & NA & Yes \\
\hline Matos et al., 2019 & VEN & Sprague-Dawley & $\mathrm{M}$ & NA & $280-300 \mathrm{~g}$ & NA & Yes \\
\hline Mulinari-Santos et al., 2016 & BRA & Wistar & $\mathrm{M}$ & 16 weeks & $250-300 \mathrm{~g}$ & 16 & Yes \\
\hline Santos et al., 2015 & BRA & Wistar & $\mathrm{M}$ & $50-64 \mathrm{~d}$ & $196-270 \mathrm{~g}$ & 20 & Yes \\
\hline \multicolumn{8}{|c|}{ Animal model: Mouse } \\
\hline Reference & Country & Strain/animal & Sex & Age & Weight & $\begin{array}{l}\text { Total number: } \\
\text { control and } \\
\text { intervention }\end{array}$ & $\begin{array}{c}\text { Ethics } \\
\text { committee }\end{array}$ \\
\hline Gonçalves-Zillo et al., 2013 & BRA & C57BL/6 & $\mathrm{M}$ & 12 weeks & NA & 16 & Yes \\
\hline Li et al., 2019 & $\mathrm{CHN}$ & Nos3 ${ }^{--}$ & $\mathrm{F}$ & 7-8 weeks & NA & 30 & Yes \\
\hline Moura et al., 2016 & BRA & C57BL/6J & $\mathrm{M}$ & 10 weeks & NA & 40 & Yes \\
\hline Oliveira et al., 2019 & BRA & $\mathrm{Balb} / \mathrm{c}$ & $\mathrm{M}$ & NA & $30 \pm 5 \mathrm{~g}$ & 12 & Yes \\
\hline Queiroz-Junior et al., 2015 & BRA & C57BL/6J and Balb/c & M & NA & NA & 20 & Yes \\
\hline Suda et al., 2013 & JPN & NA & M & 6 weeks & NA & 16 & Yes \\
\hline
\end{tabular}


Table 3. Qualitative synthesis of study characteristics and outcomes. ?: only significant results are presented. *: InterventionA: treatment started on the same day as EP induction; InterventionB: animals previously treated with Losartan for $30 \mathrm{~d}$ followed by induction of EP for $14 \mathrm{~d}$. \#: control and intervention with primary hypertension as baseline characteristic; i.d.w. $=$ in drinking water. \%: compression and tension sites were investigated separately after $6 \mathrm{~d}$ and $12 \mathrm{~d}$ for histopathologic assessment and after $0 \mathrm{~h}$ and $12 \mathrm{~h}$ for molecular assessment; outcomes reported are shown when appeared in either compression or tension site. +: mice and rats with matches control groups; i.d.w. = in drinking water.

\begin{tabular}{|c|c|c|c|c|c|c|c|c|c|c|c|c|c|c|c|c|}
\hline 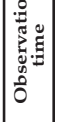 & 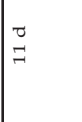 & 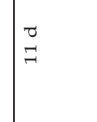 & $\stackrel{\nabla}{\exists}$ & 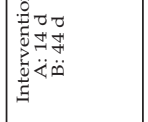 & $\vec{\pi}$ & 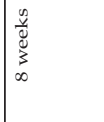 & $i_{1}$ & $I^{2}$ & $\stackrel{\pi}{\Lambda}$ & $I_{1}$ & $\pi$ & 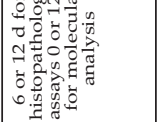 & గ్ర & $I_{\text {In }}^{0}$ & 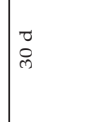 & $\underset{\mp}{\tau}$ \\
\hline & 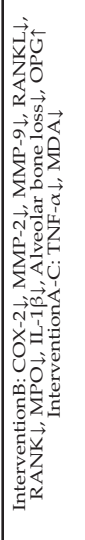 & 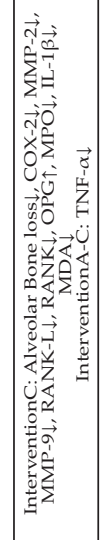 & 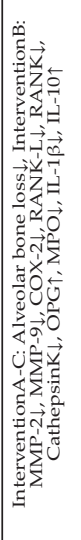 & 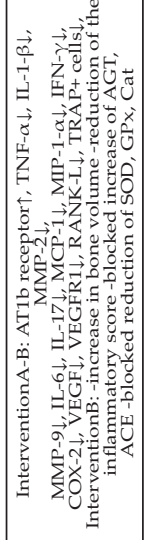 & 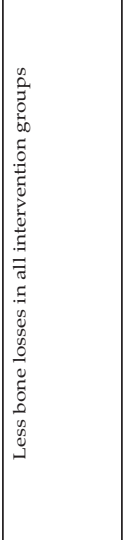 & 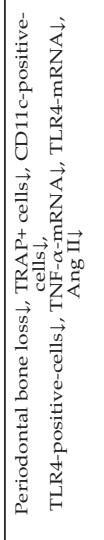 & $\begin{array}{l}\vec{x} \\
\frac{1}{\Sigma}\end{array}$ & 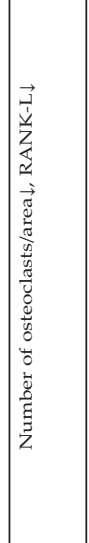 & 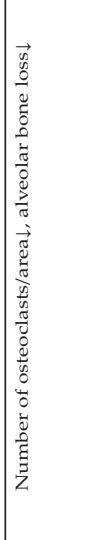 & $\begin{array}{l}\overrightarrow{\vec{v}} \\
\vec{H} \\
\overrightarrow{\vec{z}} \\
\underline{\vec{g}}\end{array}$ & $\overrightarrow{\tilde{\tilde{g}}}$ & 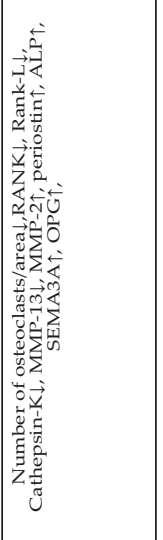 & & 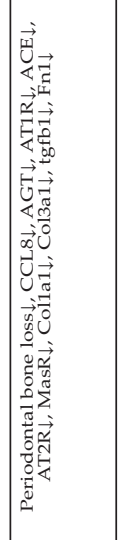 & 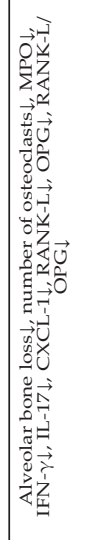 & 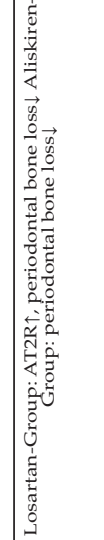 \\
\hline & 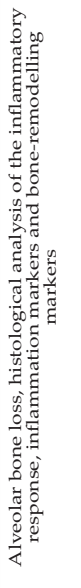 & 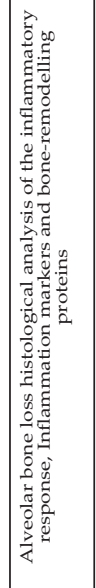 & 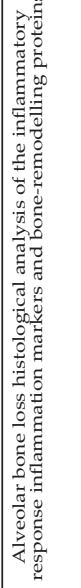 & 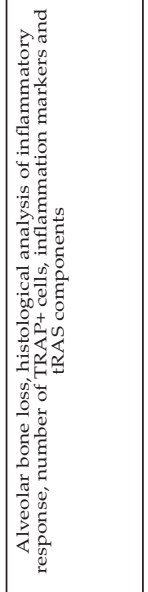 & 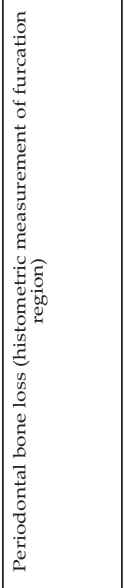 & 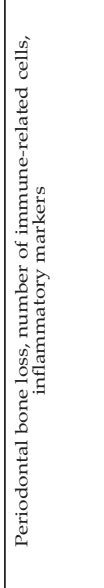 & 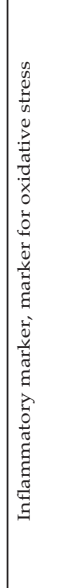 & 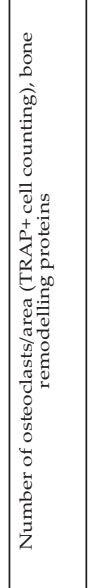 & 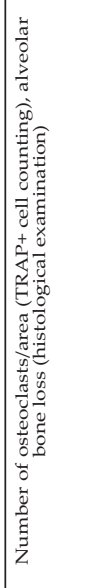 & 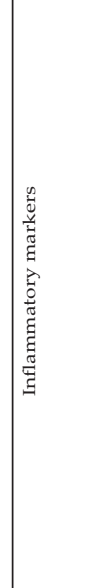 & 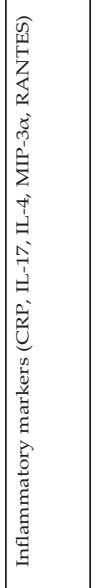 & 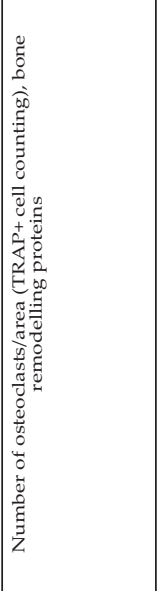 & 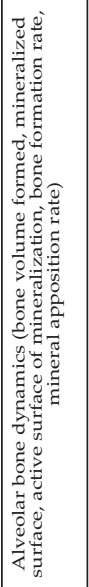 & 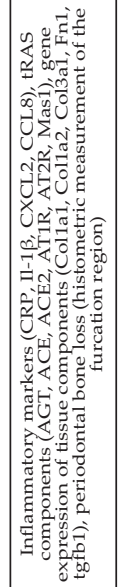 & 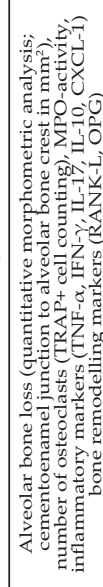 & 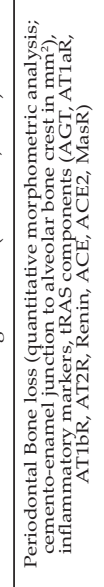 \\
\hline |ृ: & 墕 & 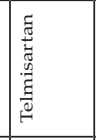 & 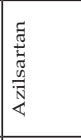 & 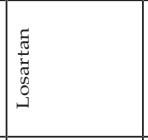 & 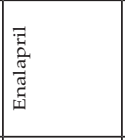 & 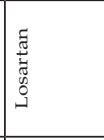 & 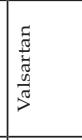 & 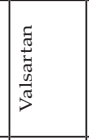 & $\mid$ & \begin{tabular}{|l}
$\frac{5}{5}$ \\
$\frac{5}{50}$ \\
$\frac{5}{5}$
\end{tabular} & 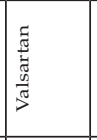 & 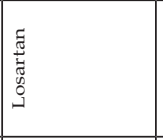 & 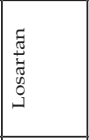 & 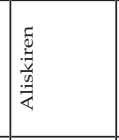 & 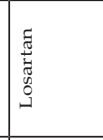 & 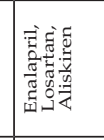 \\
\hline & 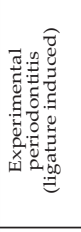 & 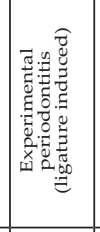 & 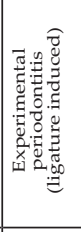 & 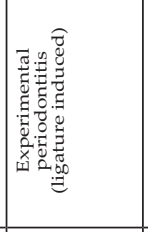 & 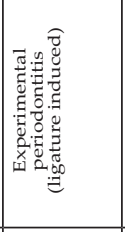 & 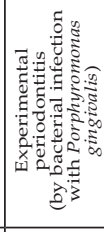 & 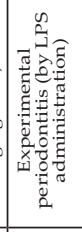 & 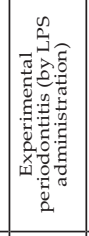 & 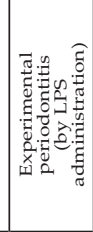 & 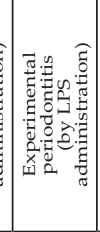 & 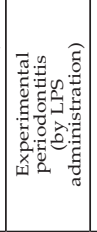 & 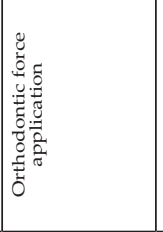 & & 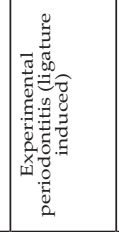 & 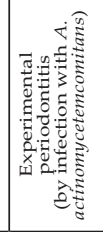 & 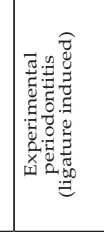 \\
\hline & 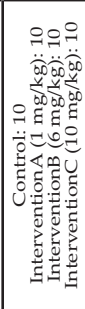 & 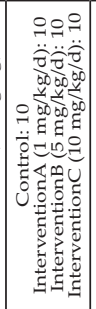 & 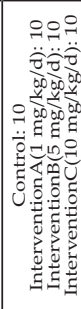 & 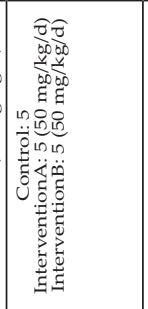 & 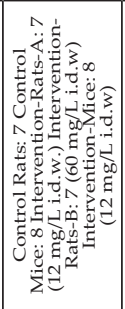 & 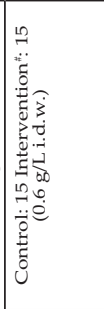 & 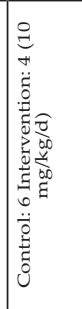 & 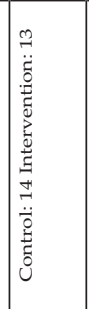 & 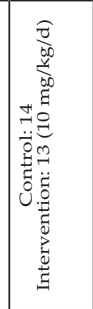 & 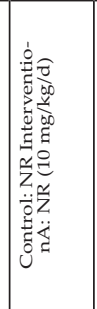 & 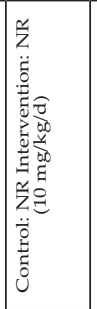 & 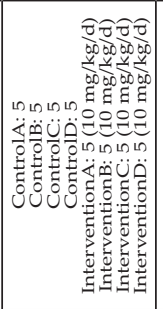 & 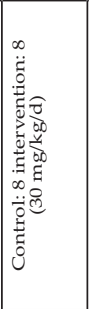 & 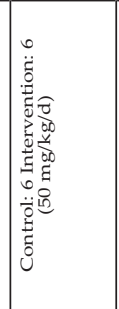 & 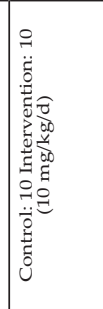 & 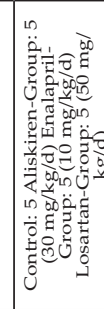 \\
\hline | & 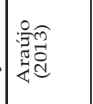 & 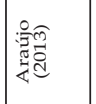 & 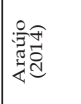 & $\frac{5}{0}$ & 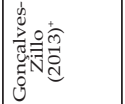 & 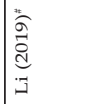 & 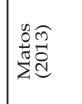 & 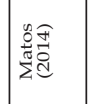 & 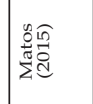 & 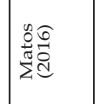 & 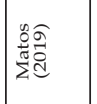 & 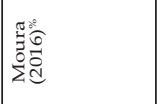 & 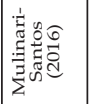 & 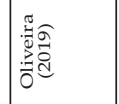 & 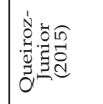 & 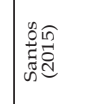 \\
\hline
\end{tabular}


$100 \mu \mathrm{L}\left(5 \times 10^{9}\right.$ cells $\left./ \mathrm{mL}\right)$ by oral and anal topical application (Li et al., 2019). In contrast, all animals in the study conducted by Suda et al. were treated with antibiotics for $10 \mathrm{~d}$ (sulphamethoxazole trimethoprim at $0.08-0.016 \%$ in the drinking water ad libitum) and were infected with the Porphyromonas gingivalis strain $\left(10^{9}\right.$ colony-forming units in $0.2 \mathrm{~mL}$ of phosphatebuffered saline and $2 \%$ carboxymethylcellulose) by gavage on the maxillary gingiva (Suda et al., 2013). The orthodontic force application method was conducted by bonding a nickel-titanium coil spring by light-cured resin between the maxillary first molar and both maxillary incisors. The apparatus was then calibrated to exert a force of approximately $0.34 \mathrm{~N}$ (Moura et al., 2016). Endotoxin-induced periodontitis was accomplished by injections of $10 \mu \mathrm{L}(1 \mathrm{mg} / \mathrm{mL})$ chromatographically purified Escherichia coli LPS into the gingiva. The pharmacological intervention arm ARB was used by 15 studies, both ACE-inhibitors and renin-inhibitors were used by 2 studies. One study compared all tRAS inhibitors in the experimental setup. Aliskiren was examined solely as a renininhibitor, whereas Enalapril was investigated as ACEinhibitor. For the ARB class, Olmesartan, Telmisartan, Azilsartan, Valsartan and Losartan were examined. Three studies (Araujo et al., 2013b; Araújo et al., 2014) investigated the dose-dependent effects of the ARBs and 1 study (Goncalves-Zillo et al., 2013) the dosedependent effect of ACE-inhibitor on the outcomes of interest. The duration of the experiments ranged from $12 \mathrm{~h}$ to $67 \mathrm{~d}$, with 11 studies reporting results between $12 \mathrm{~h}$ and $15 \mathrm{~d}$ and 6 studies reporting results between $21 \mathrm{~d}$ and $67 \mathrm{~d}$.

One of the 2 intervention groups examined by Dionisio et al. had previously been treated with Losartan for $30 \mathrm{~d}$ before induction of experimental periodontitis. This was also included in our synthesis to examine the effects on the outcomes of interest (Dionisio et al., 2019). Li et al. examined these outcomes in primary hypertensive mice, in both the intervention and matched control group ( $\mathrm{Li}$ et al., 2019). Moura et al. examined them separately at tension and compression sites, after orthodontic force application in separate intervention groups and matched control groups, the intervention groups being evaluated as separate experiments (Moura et al., 2016).

\section{Qualitative synthesis of the primary outcome $A R B s$ and periodontal bone loss}

Three studies conducted by Araujo et al. examined the impact of different ARBs (Olmesartan, Telmisartan and Azilsartan), with different doses in the experimental ligature induced periodontitis setup, on periodontal bone loss as measured by digital photography and image analysis software (Araujo et al., 2013a; Araujo et al., 2013b; Araújo et al., 2014). Bone loss in the Olmesartan group, with doses of $6 \mathrm{mg} / \mathrm{kg} / \mathrm{d}$, was significantly reduced compared to the untreated ligated group $(4.03 \pm 0.17$ vs. $7.02 \pm 0.17 \mathrm{~mm})$. The Olmesartan groups - with doses of $1 \mathrm{mg} / \mathrm{kg} / \mathrm{d}$ and
$10 \mathrm{mg} / \mathrm{kg} / \mathrm{d}$, respectively - also showed lower bone losses; however, these findings were not significant. With $10 \mathrm{mg} / \mathrm{kg} / \mathrm{d}$ Telmisartan, bone loss was significantly lower compared to the untreated ligated group ( $4.1 \pm 0.8$ vs. $7.02 \pm 0.17 \mathrm{~mm})$. The Telmisartan groups with doses of $1 \mathrm{mg} / \mathrm{kg} / \mathrm{d}$ and $5 \mathrm{mg} / \mathrm{kg} / \mathrm{d}$ also showed lower bone losses; however, these findings were not significant. With $5 \mathrm{mg} / \mathrm{kg} / \mathrm{d}$ Azilsartan, bone loss was significantly lower compared to the untreated ligated group $(2.5 \pm 1.9$ vs. $4.6 \pm 1.4 \mathrm{~mm})$. The Azilsartan groups with doses of $1 \mathrm{mg} / \mathrm{kg} / \mathrm{d}$ and $10 \mathrm{mg} / \mathrm{kg} / \mathrm{d}$, respectively, also showed lower bone losses; however, these findings were not significant. Dionisio et al. examined the impact of Losartan on bone volume, after experimental ligature induced periodontitis in two intervention groups, as measured by computer tomography (Dionisio et al., 2019). One of the intervention groups was pre-treated with Losartan $(50 \mathrm{mg} / \mathrm{kg} / \mathrm{d})$ for $30 \mathrm{~d}$ before and for a further $14 \mathrm{~d}$ after induction of experimental periodontitis, and one group was treated with Losartan $(50 \mathrm{mg} /$ $\mathrm{kg} / \mathrm{d}$ ) simultaneously with experimental periodontitis induction, lasting $14 \mathrm{~d}$ from then on. The intervention group with Losartan treatment, simultaneous to experimental periodontitis induction, showed higher bone volumes after $14 \mathrm{~d}$; however, this finding was not significant. The intervention group with Losartan pre-treatment showed significantly higher bone volumes after $14 \mathrm{~d}$ compared to the untreated group $\left(65.08 \pm 6.92\right.$ vs. $\left.37.4 \pm 8.31 \mathrm{~cm}^{3}\right)$. Li et al. examined the impact of Losartan on periodontal bone loss, as measured by digital microscopy and an image analysis software, in an experimental periodontitis model induced by bacterial infection with Porphyromonas gingivalis (Li et al., 2019). Both intervention and matched control group had primary hypertension as a shared baseline characteristic. Treatment with Losartan resulted in significantly lower bone loss measurements when compared to the untreated group $\left(0.82 \pm 0.18\right.$ vs. $\left.1.83 \pm 0.1 \mathrm{~mm}^{2}\right)$. In the same study, primary hypertensive mice without experimental periodontitis showed higher, but not significant, bone resorption compared to the non-hypertensive mice and this could also not be reduced through Losartan application. Thus, the lower bone losses through Losartan applications were only seen in the experimental periodontitis groups. Matos et al. examined the impact of Valsartan in rats with periodontitis, induced by four injections of LPS with Escherichia coli, within the experimental period of $7 \mathrm{~d}$ (Matos et al., 2015). Periodontal bone loss was measured by digital microscopy and quantification of density histograms. The Valsartan treated group showed lower bone loss compared to the untreated group. Interestingly, the Valsartan treated group also showed higher bone densities when compared to the non-periodontitis group. However, no data regarding statistical significance was provided; therefore, the provided data could not be included in the quantitative synthesis and only the qualitative results of the findings were included. Mulinari- 
Santos et al. examined the impact of Losartan on bone volumes in normal and spontaneously hypertensive rats with each having matched control groups (Mulinari-Santos et al., 2019). Quantitative measurements were made with confocal laser scanning microscopy and image analysis software. The bone volume formed increased significantly in the Losartan treated group as compared to the untreated normal rat group $(0.071 \pm 0.01$ vs. $0.039 \pm 0.003 \mathrm{~mm}^{3} / \mathrm{d}$ ). The increase in bone volume in the spontaneously hypertensive rats treated with Losartan, when compared to the untreated, was not significant. However, Losartan treated groups also showed a statistically significant increase in the mineralised surface, active surface of mineralisation, bone formation rate, and mineral apposition rate in the alveolar bone, as measured by biomarking of fluorochromes. Queiroz-Junior et al. examined the impact of Losartan on periodontal bone loss in an experimental periodontitis mouse model induced by palatal injection of $A$. actinomycetemcomitans suspension (Queiroz-Junior et al., 2015). Bone loss was quantified by digital photography and morphometric analysis with image analysis software. The Losartan treated group showed significantly lower bone losses after $30 \mathrm{~d}$ of experimental periodontitis as compared to the untreated group $(0.297 \pm 0.134$ vs. $0.494 \pm 0.249 \mathrm{~mm}^{2}$ ).

\section{ACE-inhibitor and periodontal bone loss}

Goncalves-Zillo et al. examined the impact of Enalapril, an ACE-inhibitor, on periodontal bone loss, as measured by histometric evaluation of the furcation region after $21 \mathrm{~d}$, in an experimental ligature induced periodontitis model, in both rats and mice with matched control groups (GoncalvesZillo et al., 2013). The rat intervention group, with an application of $12 \mathrm{mg} / \mathrm{L}$ Enalapril in drinking water for $21 \mathrm{~d}$, showed significantly lower bone losses compared to the untreated control group $(0.23 \pm 0.12$ vs. $\left.0.4 \pm 0.02 \mathrm{~mm}^{2}\right)$. The rat intervention group, with $16 \mathrm{mg} / \mathrm{L}$, also showed significantly lower bone losses compared to the control group $(0.25 \pm 0.09$ vs. $\left.0.4 \pm 0.02 \mathrm{~mm}^{2}\right)$. However, the group with $16 \mathrm{mg} / \mathrm{L}$ Enalapril, but not the group with $12 \mathrm{mg} / \mathrm{L}$ in drinking water, showed a significant reduction in systolic blood pressure; thus, comparability to the matched group is only given for the $12 \mathrm{mg} / \mathrm{L}$ group. In mice, only $12 \mathrm{mg} / \mathrm{L}$ Enalapril was added to the drinking water and resulted in significantly lower bone losses after $21 \mathrm{~d}$ as compared to the untreated group $\left(101.66 \pm 15.33\right.$ vs. $\left.129.67 \pm 15.33 \mathrm{~mm}^{2}\right)$.

\section{Renin-inhibitor and periodontal bone loss}

Oliveira et al. examined the impact of Aliskiren on periodontal bone loss in an experimental ligature induced periodontitis mouse model (Oliveira et al., 2019). Bone loss was measured by morphometric measurements of the furcation region using image analysis software. The experiments were conducted in two intervention groups, normal and diabetic mice, with matched control groups. Aliskiren treated mice group showed a significantly higher percentage of bone area per $\mu \mathrm{m}^{2}\left(\% / \mu \mathrm{m}^{2}\right)$ compared to the untreated normal control group (35.08 \pm 3.31 vs. $24.24 \pm 1.05 \%$ / $\mu \mathrm{m}^{2}$ ). This significant increase of alveolar bone area was also seen in the Aliskiren group of diabetic mice compared to the matched but untreated control group.

Comparison of different $t R A S$ inhibitors and periodontal bone loss

The study conducted by Santos et al. was the only included study comparing the effects of different tRAS inhibitors, Aliskiren, Enalapril and Losartan, regarding periodontal bone loss (Santos et al., 2015). Experimental periodontitis was induced by ligature and the experimental observation period lasted $14 \mathrm{~d}$. Aliskiren, Enalapril and Losartan were administered daily over the experimental period with doses of 10,30 and $50 \mathrm{mg} / \mathrm{kg}$, respectively. Bone loss measurements were conducted using digital photography and image analysis software. Bone loss in the Aliskiren treated group was significantly reduced as compared to the shared control group $\left(2.08 \pm 0.36\right.$ vs. $\left.3.29 \pm 0.55 \mathrm{~mm}^{2}\right)$. The Losartan group also showed significantly lower bone losses compared to the shared control group (2.22 \pm 0.33 vs. $3.29 \pm 0.55 \mathrm{~mm}^{2}$ ). Bone loss in the Enalapril group was also lower compared to the shared control group $\left(2.86 \pm 0.38\right.$ vs. $\left.3.29 \pm 0.55 \mathrm{~mm}^{2}\right)$; however, this finding failed to reach statistical significance.

\section{Qualitative synthesis of the secondary outcomes Bone remodelling markers}

The impact of tRAS inhibition on bone remodelling markers was analysed by 3 studies (Matos et al., 2014; Moura et al., 2016; Queiroz-Junior et al., 2015) (Table 3 and Fig. 3). All of these studies used ARBs as the pharmacological intervention arm and the time until examination ranged from $12 \mathrm{~h}$ to $30 \mathrm{~d}$. Valsartan non-significantly decreased the optical immunofluorescence density (arbitrary unit (AU) as measured by confocal microscopy and image analysis software) of RANK (5391.38 \pm 181.25 vs. $6157.99 \pm 188.09$ AU), RANKL $(6188.41 \pm 679.31$ vs. $7333.33 \pm 488.04 \mathrm{AU})$, the ratio of RANKL/OPG $(1.01 \pm 0.05$ vs. $7.35 \pm 1.07)$ and increased OPG $(5632.65 \pm 401.36$ vs. $990.72 \pm 208.26$ AU). The study conducted by Moura et al. (2016) with Losartan application in a mice model of orthodontic force application and separate examination of tension and compression sites with matched control groups showed a decrease of RANK mRNA expression (AU calculated as relative fold gene expression, normalised to $\beta$-actin as the internal control; $2^{-\Delta \Delta c t}$ method) after $12 \mathrm{~h}$ for the tension $(0.88 \pm 0.09$ vs. $1.27 \pm 0.07 \mathrm{AU})$ and compression sites $(1.24 \pm 0.09$ vs. $2.27 \pm 0.26 \mathrm{AU})$, respectively. RANKL mRNA in tension $(0.71 \pm 0.05$ vs. $0.74 \pm 0.09 \mathrm{AU})$ and compression sites $(1.87 \pm 0.09$ vs. $2.76 \pm 0.23 \mathrm{AU})$ also decreased; however, only the decrease at the compression sites was significant. 


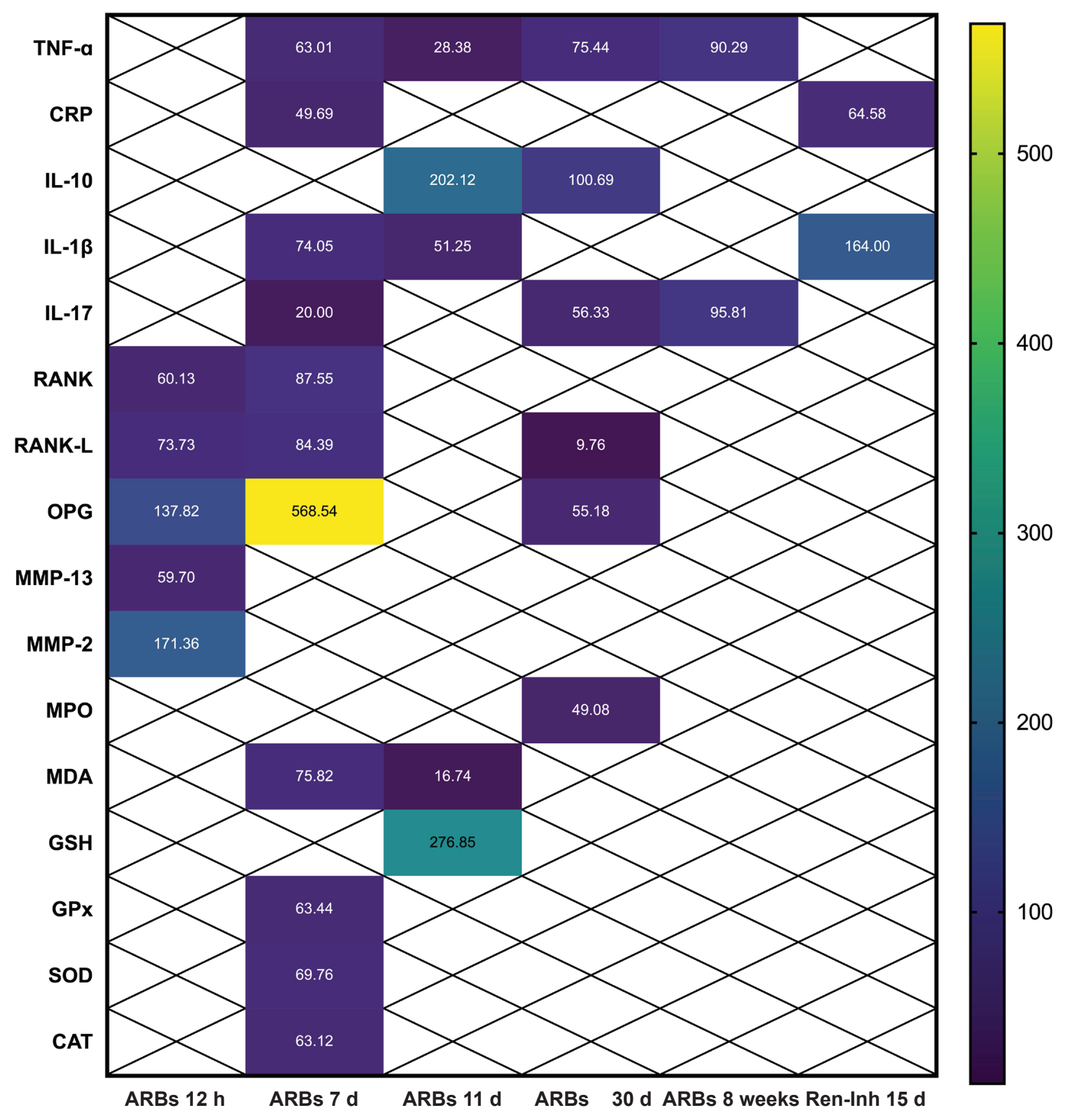

Fig. 3. Heatmap illustration of secondary outcomes. Outcomes after intervention are shown as a percentage (\%) of the reference value (untreated control group). $\mathrm{CRP}=\mathrm{c}$-reactive protein; $\mathrm{MPO}=$ myeloperoxidase; $\mathrm{MDA}=$ malondialdehyde; $\mathrm{GSH}$ = glutathione; $\mathrm{GPx}$ = glutathione peroxidase; $\mathrm{SOD}$ = superoxide dismutase; $\mathrm{CAT}=$ catalase .

Furthermore, Losartan decreased mRNA expression of cathepsin $\mathrm{K}$ for the tension $(1.17 \pm 0.11$ vs. $1.25 \pm 0.19 \mathrm{AU})$ and compression $(2.15 \pm 0.17$ vs. $3.5 \pm 0.29 \mathrm{AU})$ sites and increased OPG mRNA expression in the tension $(2.05 \pm 0.1$ vs. $1.5 \pm 0.14 \mathrm{AU})$ and compression $(2.92 \pm 0.09$ vs. $2.1 \pm 0.15 \mathrm{AU})$ sites, respectively. However, for cathepsin $\mathrm{K}$ only the tension site and for OPG only the compression site showed statistical significance. The application of Losartan for $30 \mathrm{~d}$ in the study conducted by Queiroz et al. (2015) resulted in a significant decrease of RANKL (103.00 \pm 213.76 vs. $1055.79 \pm 967.01 \mathrm{pg} / 100 \mathrm{mg}$ tissue) and the RANKL/OPG ratio $(0.06 \pm 0.09$ vs. $0.37 \pm 0.30)$ and a significant increase of OPG $(1676.59 \pm 1534.04$ vs. $3038.29 \pm 915.04 \mathrm{pg} / 100 \mathrm{mg}$ tissue).

\section{Number of osteoclasts}

The impact of ARBs on the number of tartrateresistant acid phosphatase positive (TRAP+) cells was examined by 5 studies (Li et al., 2019; Matos et al., 2014; Matos et al., 2015; Moura et al., 2016; QueirozJunior et al., 2015). The study conducted by Matos et al. reported a significantly lower number of osteoclasts/ area, measured as the number of osteoclasts per oil-immersion field (OCs/field), after Valsartan intervention and examination after $7 \mathrm{~d}$ compared to 
the untreated group $(2.54 \pm 0.51$ vs. $5.21 \pm 1.11$ OCs/ field). The study published by Matos et al. (2015), with the same experimental setup, showed similar results confirming previous data. Losartan application for 8 weeks in the study conducted by Li et al. showed a significantly lower number of osteoclasts/area (OCs/ $\left.\mathrm{mm}^{2}\right)$, compared to the untreated group $(30.79 \pm 5.73$ vs. $87.83 \pm 6.92 \mathrm{OCs} / \mathrm{mm}^{2}$ ) (Li et al., 2019). Losartan application also resulted in a significantly lower number of osteoclasts/area (shown as osteoclasts per histologically examined $5 \mu \mathrm{m}$-thick sagittal sections of the periodontal tissue; OCs/section) after $6 \mathrm{~d}(3.58 \pm 0.59$ vs. $8.18 \pm 0.54)$ and $12 \mathrm{~d}$ $(4.97 \pm 1.77$ vs. $13.74 \pm 1.39)$ in the study conducted by Moura et al. (Moura et al., 2016). Queiroz et al. reported a significantly lower number of osteoclasts/ area (measured as osteoclasts in five consecutive microscopic fields (400×) per section; OCs/section) after $30 \mathrm{~d}$ of Losartan application compared to the untreated control group $(0.55 \pm 0.53$ vs. $1.85 \pm 1.00)$ (Queiroz-Junior et al., 2015). In contrast to these findings, Suda et al. examined the appearance of osteoclasts by immunostaining of cathepsin $\mathrm{K}$ and reported a non-significant decrease of osteoclast surface/bone surface (\%) compared to the untreated group $(4.95 \pm 2.78 \%$ vs. $5.55 \pm 2.45 \%$ ) (Suda et al., 2013).

\section{(Anti-) inflammatory markers}

Ten of the included studies (Araujo et al., 2013a; Araujo et al., 2013b; Araújo et al., 2014; Dionisio et al., 2019; Li et al., 2019; Matos et al., 2014; Matos et al., 2016; Matos et al., 2019; Oliveira et al., 2019; Queiroz-Junior et al., 2015) reported at least one of the following inflammatory markers with dysregulated expression level after tRAS inhibition: TNF- $\alpha$, IL-10, IL-1 $\beta$, IL-17, CRP. Intervention with Telmisartan showed a dose-dependent reduction of TNF- $\alpha$ in the study conducted by Araujo et al. (Araujo et al., 2013a). Doses of 1, 5 and $10 \mathrm{mg} / \mathrm{kg} / \mathrm{d}$ Telmisartan for a period of of $7 \mathrm{~d}$ resulted in significantly lower TNF- $\alpha$ concentrations (pg/mL) of $1883.66 \pm 1051.17 v s$. $5052.63 \pm 2662.97,1174.52 \pm 1051.17$ vs. $5052.63 \pm 2662.97$ and $686.98 \pm 560.63$ vs. $5052 \pm 2662.97$, respectively, compared to the untreated comparator. Suda et al. provided results for Telmisartan treatment with $5 \mathrm{mg} /$ $\mathrm{kg} / \mathrm{d}$ for 8 weeks and reported non-significant lower TNF- $\alpha$ concentrations compared to the untreated group $(25.54 \pm 1.53$ vs. $28.29 \pm 3.56$ pg/mL). Similar observations were seen after Olmesartan application with doses of 1,6 and $10 \mathrm{mg} / \mathrm{kg}$ which resulted in significantly lower TNF- $\alpha$ concentrations (pg/mL) of $1104.06 \pm 722.35$ vs. $3629.44 \pm 3090.04,241.12 \pm 321.04$ vs. $3629.44 \pm 3090.03$, and $482.23 \pm 963.13$ vs. $3629.44 \pm 3090.04$, respectively (Araujo et al., 2013b). However, the lowest concentrations of TNF- $\alpha$ were seen in the group treated with $6 \mathrm{mg} / \mathrm{kg}$ Olmesartan. For Azilsartan the lowest TNF- $\alpha$ concentrations were seen in the Azilsartan group treated with $5 \mathrm{mg} / \mathrm{kg}(755.04 \pm 674.38$ vs. $1262.25 \pm 637.92 \mathrm{pg} / \mathrm{mL})$ (Araújo et al., 2014). Furthermore, the Azilsartan groups treated with $1 \mathrm{mg} / \mathrm{kg}(933.72 \pm 947.77$ vs. $1262.25 \pm 637.92 \mathrm{pg} / \mathrm{mL})$ and $10 \mathrm{mg} / \mathrm{kg}(1204.61 \pm 856.64$ vs. $1262.25 \pm 637.92 \mathrm{pg} / \mathrm{mL}$ ) also showed lower TNF- $\alpha$ concentrations, but none of these findings reached statistical significance. In the study conducted by $\mathrm{Li}$ et al., the Losartan treated group showed significantly lower TNF- $\alpha$ concentrations after 8 weeks compared to the untreated group $(18.57 \pm 1.92$ vs. $49.53 \pm 5.75 \mathrm{pg} /$ $\mathrm{mL}$ ) (Li et al., 2019). Similar to this, Queiroz-Junior et al. reported significantly lower TNF- $\alpha$ concentrations for the Losartan group after $30 \mathrm{~d}$ (307.77 \pm 219.87 vs. $407.98 \pm 148.74 \mathrm{pg} / 100 \mathrm{mg}$ tissue) (Queiroz-Junior et al., 2015). For Valsartan, data were available from Matos et al. who reported non-significant lower TNF- $\alpha$ concentrations after $7 \mathrm{~d}$ of Valsartan treatment compared to the untreated group.

Data regarding IL-1 $\beta$ concentrations after ARB treatment with Olmesartan, Telmisartan and Azilsartan for $7 \mathrm{~d}$ were provided by Araujo et al. (Araujo et al., 2013a; Araujo et al., 2013b; Araújo et al., 2014). For Olmesartan, the lowest concentrations were seen in the group treated with $6 \mathrm{mg} / \mathrm{kg}$ Olmesartan (56.45 \pm 32.26 vs. $786.29 \pm 177.42$ pg/ $\mathrm{mL}$ ). The Olmesartan groups treated with 1 and $6 \mathrm{mg} / \mathrm{kg}$, respectively, also showed lower IL-1 $\beta$ concentrations. However, only the findings for $6 \mathrm{mg} / \mathrm{kg}$ Olmesartan reached statistical significance. For Telmisartan, the $10 \mathrm{mg} / \mathrm{kg}$ treated Telmisartan group showed the significantly lowest IL-1 $\beta$ concentrations $(137.54 \pm 203.87$ vs. $876.79 \pm 448.52 \mathrm{pg} /$ $\mathrm{mL})$. Telmisartan groups with 1 and $6 \mathrm{mg} / \mathrm{kg}$ also showed lower IL-1 $\beta$ levels, but these findings did not reach statistical significance. For Azilsartan, lower IL-1 $\beta$ concentrations were only seen in the $1 \mathrm{mg} / \mathrm{kg}(666.67 \pm 121.11$ vs. $1217.63 \pm 165.29 \mathrm{pg} / \mathrm{mL})$ and the $5 \mathrm{mg} / \mathrm{kg}$ Azilsartan group (512.4 \pm 104.68 vs. $1217.63 \pm 165.29 \mathrm{pg} / \mathrm{mL}$ ), with only the latter reaching statistical significance. The IL-1 $\beta$ concentration of the $10 \mathrm{mg} / \mathrm{kg}$ Azilsartan group was not significantly different from the untreated control group. Matos et al. reported non-significant lower IL-1 $\beta$ concentrations after $7 \mathrm{~d}$ of treatment with Valsartan (Matos et al., 2016). Oliveira et al. was the only study reporting that IL-1 $\beta$ concentration after Aliskiren treatment, and IL-1 $\beta$ concentration after Aliskiren treatment for $14 \mathrm{~d}$, was not significantly different compared to the untreated control group (Oliveira et al., 2019).

For IL-10, an anti-inflammatory marker, data were provided by 4 studies (Araujo et al., 2013a; Araujo et al., 2013b; Araújo et al., 2014; Queiroz-Junior et al., 2015). For Olmesartan and Telmisartan, treatment for $7 \mathrm{~d}$ with concentrations of 1,6 and $10 \mathrm{mg} / \mathrm{kg}$ increased IL-10 concentrations in all treated groups (Araujo et al., 2013a; Araujo et al., 2013b). However, none of the findings reached statistical significance. For Azilsartan, the group treated with $5 \mathrm{mg} / \mathrm{kg}$ Azilsartan showed significantly higher IL-10 levels compared to the untreated control group (3207.76 \pm 3311.19 vs. $814.40 \pm 473.03 \mathrm{pg} / \mathrm{mL}$ ) (Araújo et al., 2014). The groups treated with 1 and $10 \mathrm{mg} / \mathrm{kg}$, respectively, were not significantly different from the untreated 
control group. Treatment with Losartan $(10 \mathrm{mg} / \mathrm{kg} / \mathrm{d})$ for $30 \mathrm{~d}$ resulted in no significant difference in IL-10 levels compared to the control group (Queiroz-Junior et al., 2015).

Data regarding IL-17 concentrations after inhibition with ARBs were provided by 3 studies (Matos et al., 2019; Oliveira et al., 2019; Suda et al., 2013). Treatment with Losartan for $30 \mathrm{~d}$ resulted in a significant reduction of IL-17 levels compared to the untreated control group (87.63 \pm 54.36 vs. $155.56 \pm 45.08 \mathrm{pg} / 100 \mathrm{mg}$ tissue). Valsartan treatment for $7 \mathrm{~d}$ showed no significant reduction of IL-17 levels compared to the control group (Matos et al., 2019). Further, treatment with $5 \mathrm{mg} / \mathrm{kg}$ Telmisartan for 8 weeks showed no significant differences compared to the untreated control group (Suda et al., 2013).

CRP concentrations were examined after $15 \mathrm{~d}$ of treatment with Aliskiren (Oliveira et al., 2019). CRP concentrations were non-significantly lower in the treatment group, compared to the control group (1957.49 \pm 447.43 vs. $3031.32 \pm 380.31$ pg/mL). For ARBs, data was available for Valsartan which led to significantly lower CRP concentrations after $7 \mathrm{~d}$ compared to the untreated control group (Matos et al., 2016; Matos et al., 2019).

\section{Oxidative stress markers}

Myeloperoxidase (MPO), malondialdehyde (MDA), superoxide dismutase (SOD), catalase (CAT), glutathione peroxidase (GPx), and glutathione (GSH) as markers for oxidative stress were only examined after inhibition with ARBs (Araujo et al., 2013a; Araujo et al., 2013b; Araújo et al., 2014; Matos et al., 2013; Queiroz-Junior et al., 2015). Olmesartan treatment resulted in a significant reduction of MDA, regardless of the dose (Araujo et al., 2013b). For MPO, all doses also resulted in lower levels, but only the $6 \mathrm{mg} / \mathrm{kg}$ Olmesartan group reached statistical significance $(0.8 \pm 1.61$ vs. $23.74 \pm 11.28 \mathrm{nmol} / \mathrm{g}$ tissue $)$. GSH levels in all Olmesartan groups were not significantly different from the control group. Similar findings were found for Telmisartan and levels of MPO, MDA, and GSH (Araujo et al., 2013a). However, only the $10 \mathrm{mg} / \mathrm{kg}$ resulted in significantly lower MDA and MPO levels. Higher levels of GSH were found for the different Telmisartan groups, but none of these findings reached statistical significance. Also, all doses of Azilsartan resulted in lower MPO levels, but only the $5 \mathrm{mg} / \mathrm{kg}$ Azilsartan group reached statistical significance (3.01 \pm 1.32 vs. $7.54 \pm 11.44 \mathrm{nmol} / \mathrm{g}$ tissue) (Araújo et al., 2014). For GSH levels after Azilsartan treatment, non-significant higher GSH levels were found in the different Azilsartan groups. Valsartan treatment for $7 \mathrm{~d}$ resulted in significantly lower MDA levels compared to the control group (31.29 \pm 1.04 vs. $41.27 \pm 2.04 \mathrm{nmol} / \mathrm{mL})$. Furthermore, Valsartan resulted in non-significant lower levels of SOD, CAT and GPx compared to the untreated control group. Queiroz et al. reported significantly lower MPO levels for the Losartan treated group, compared to the control group (Queiroz-Junior et al., 2015).

\section{Quantitative synthesis of the primary outcome}

Presentation of the network geometry

Network geometry of the control group and the tRAS inhibitor classes are illustrated in Fig. 4. Four interventions were compared in this network geometry (control, ARB, ACE-inhibitor, and renin-inhibitor). Sixteen comparisons between ARBs and control, 4 comparisons between ACE-inhibitors and control, 2 comparisons between renin-inhibitor and control, 1 comparison between ARB and renin-inhibitor, 1 comparison between ARB and ACE-inhibitor and 1 comparison between renin-inhibitor and ACE inhibitor were considered. The network geometry for the different doses of tRAS inhibitors is shown in Fig. 6. Closed loops were present for Olmesartan, Telmisartan and Azilsartan (each with three different doses), Losartan and "PreLosartan" (treatment of Losartan before induction of experimental periodontitis), Enalapril (with 2 different doses) and the cross-comparison between Aliskiren, Losartan and Enalapril. Enalapril12mg, Enalapril60mg and Losartan600mg were given as $\mathrm{mg} / \mathrm{L}$ in drinking water for the observation time. Because the authors did not provide the weight of the animals, no estimation of the values in $\mathrm{mg} / \mathrm{kg} / \mathrm{d}$ could be performed. All other doses are given in $\mathrm{mg} / \mathrm{kg} / \mathrm{d}$ during the period of the experiments.

\section{Network interval plot and treatment ranks}

Network interval plot and treatment ranks are shown in Fig. 5. There was a statistically significant difference between $\mathrm{ARB}$ vs. control $(\mathrm{SMD}=-1.26$, $95 \% \mathrm{CI}-1.93$ to -0.58$)$, ACE-inhibitor vs. control $(\mathrm{SMD}=-1.43,95 \% \mathrm{CI}-2.74$ to -0.12$)$ and renininhibitor $v s$. control (SMD $=-2.11,95 \% \mathrm{CI}-3.85$ to - 0.37). Thus, all tRAS inhibitor classes resulted in lower periodontal bone loss compared to the control group in this analysis. Furthermore, no significant differences were found when comparing the different tRAS inhibitors on periodontal bone loss. According to the SUCRA ranking, the most effective tRAS inhibitor with regards to preventing periodontal bone loss was the renin-inhibitor (Aliskiren). ARBs and ACE-inhibitor (Enalapril) performed similarly and the worst was the untreated control group.

\section{Network forest plot for the different $t R A S$ doses}

Network forest plot for the different tRAS doses is shown in Fig. 7. Statistically significant differences in SMD were seen for Aliskren30mg vs. control $(\mathrm{SMD}=-2.13,95 \% \mathrm{CI}-3.74$ to -0.52$)$, Aliskren50mg vs. control (SMD $=-1.64,95 \% \mathrm{CI}-3.21$ to -0.58$)$, Enalapril12mg vs. control (SMD $=-1.98,95 \% \mathrm{CI}-3.02$ to -0.94$)$, Enalapril60mg vs. control $(\mathrm{SMD}=1.71$, $95 \%$ CI -3.01 to -0.42$)$, Losartan30mg vs. control $(\mathrm{SMD}=-3.88,95 \% \mathrm{CI}-5.86$ to -1.91$)$, Losartan $50 m g$ vs. control $(\mathrm{SMD}=-1.5,95 \% \mathrm{CI}-2.65$ to -0.35$)$, Losartan600mg vs. control (SMD $=-6.75,95 \%$ CI -8.53 to -4.64$)$, PreLosartan50mg vs. control $(\mathrm{SMD}=-1.59,95 \% \mathrm{CI}-3.03$ to -0.14$)$. Overall, most of the intervention groups performed better than the 
control group and Losartan600mg group performed significantly better than all other comparators. In addition, PreLosartan50mg performed better than the control group but SMD was not significantly different when comparing to other tRAS inhibitor groups. Furthermore, significant differences were found comparing Azilsartan10mg vs. Aliskren30mg $(\mathrm{SMD}=2.27,95 \%$ CI 0.28 to 4.26$)$, Losartan600mg vs.

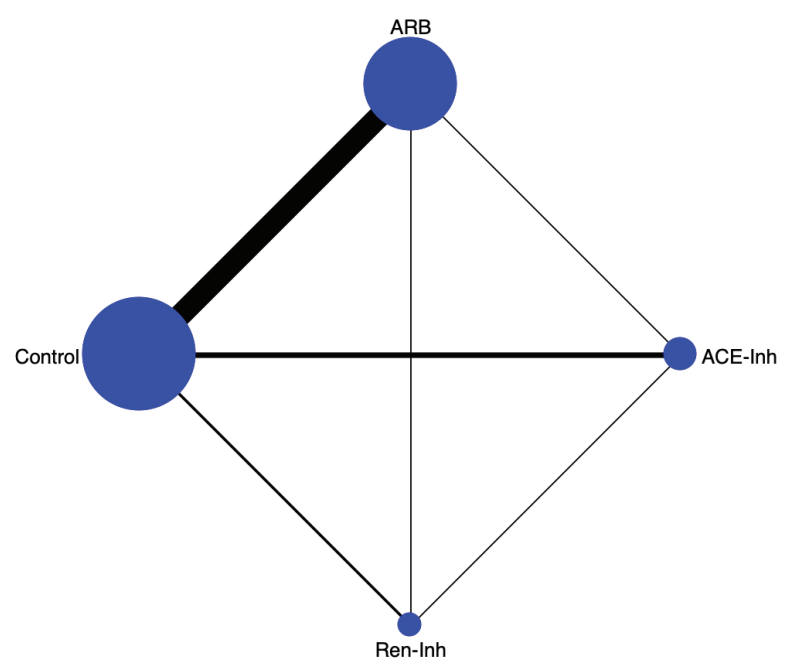

Fig. 4. Network geometry for inhibitor types and the outcome periodontal bone loss. Nodes represent the inhibitor types and edges represent the comparisons. The thickness of lines and nodes represent the number of reporting studies. ARB = Angiotensin II type 1 receptor blocker; ACE-Inh = Angiotensin-Converting-Enzyme inhibitor; RenInh $=$ Renin inhibitor .
Aliskren30mg (SMD $=-4.62,95 \% \mathrm{CI}-7.27$ to -1.97$)$, Losartan600mg vs. Aliskren50mg (SMD = -5.11, $95 \%$ CI -7.74 to -2.48$)$, Azilsartan6mg vs. Azilsartan10mg $(\mathrm{SMD}=-1.25,95 \% \mathrm{CI}-2.45$ to -0.44$)$, Enalapril12mg vs. Azilsartan10mg (SMD $=-2.13,95 \% \mathrm{CI}-3.69$ to -0.56 ), Enalapril 60mg vs. Azilsartan10mg (SMD $=-1.86,95 \%$ CI - 3.59 to - 0.12), Losartan30mg vs. Azilsartan10mg $(\mathrm{SMD}=-4.03,95 \% \mathrm{CI}-6.32$ to -1.73$)$, Losartan $50 m g$ vs.

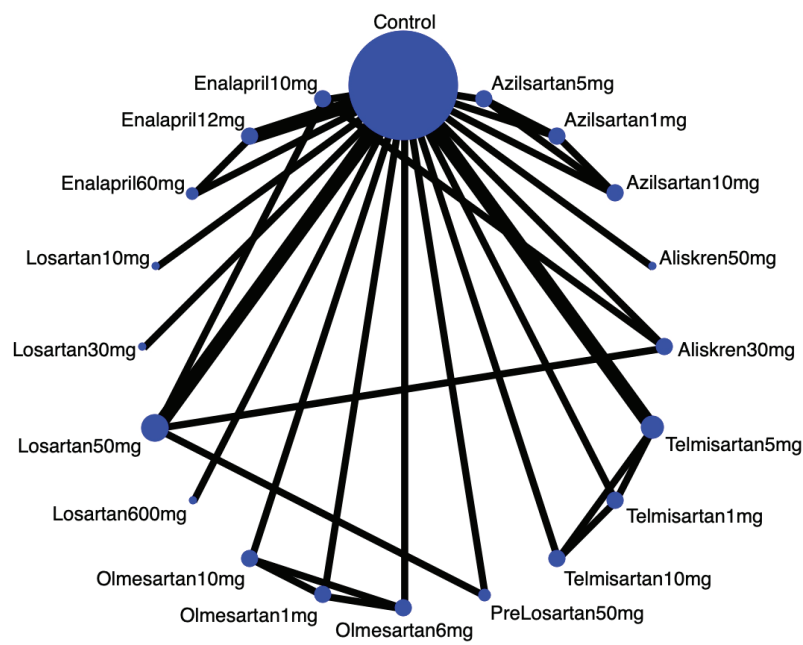

Fig. 6. Network geometry for inhibitor doses and the outcome periodontal bone loss. Nodes represent the inhibitor types and doses and edges represent the comparisons. The thickness of lines and nodes represent the number of reporting studies. Losartan600mg, Enalapril12mg, and Enalapril60mg are given as $\mathrm{mg} / \mathrm{L}$ in drinking water for the duration of the experiment. All other values are shown as $\mathrm{mg} /$ $\mathrm{kg} / \mathrm{d}$ for the duration of the experiment.

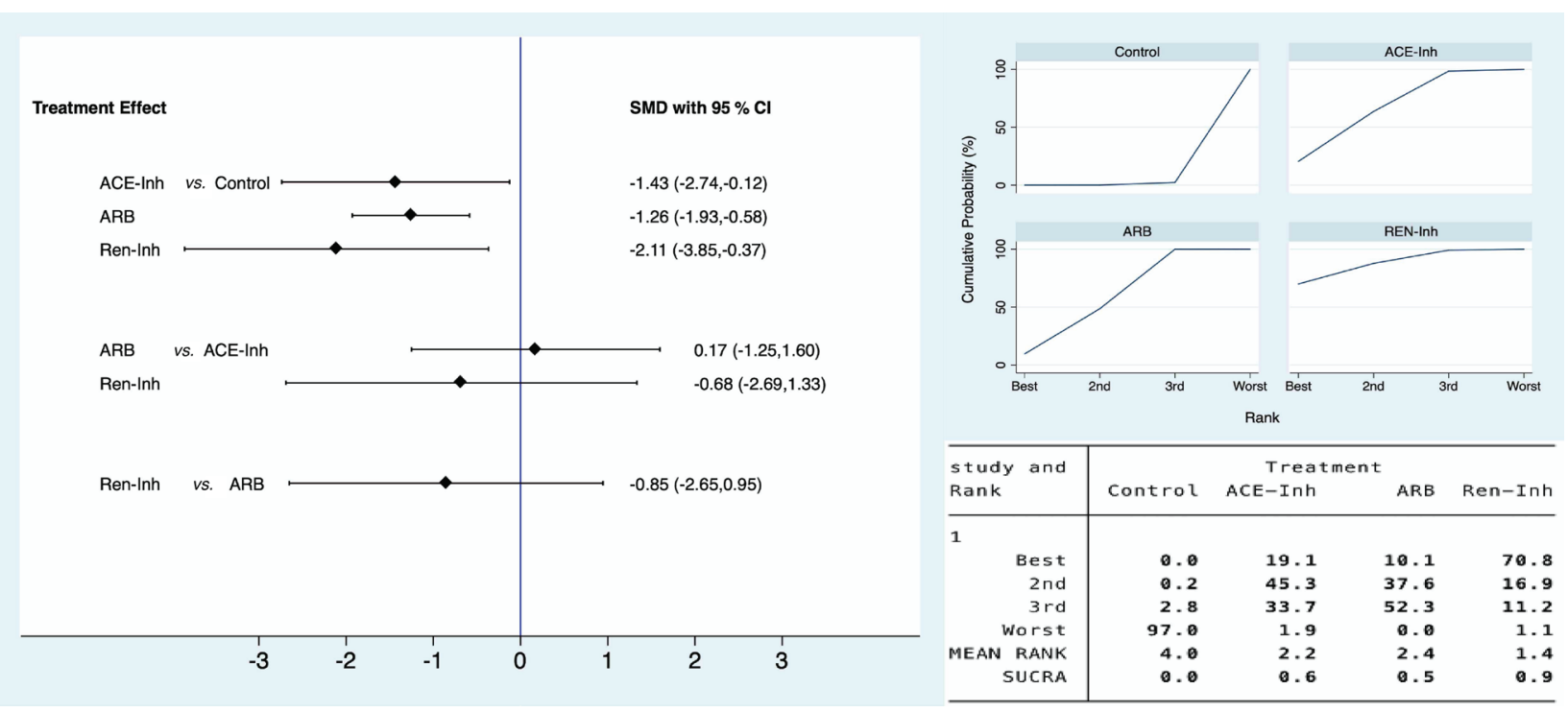

Fig. 5. Network interval plot showing the treatment effect of tRAS inhibitors and the comparator. The effect size (SMD) is shown with its $95 \%$ confidence interval. Angiotensin II type 1 receptor blockers (ARB), angiotensin-converting-enzyme inhibitors (ACE-Inh) and renin inhibitors (Ren-Inh) performed significantly better than the untreated comparator (SMD lower than 0 ("line of null effect") favours the intervention arm), resulting in lower periodontal bone losses. SUCRA plot and treatment rankings are showing the probability of a given treatment (or control) to be best, second, third, and worst in preventing periodontal bone loss. (x-axis: ranking of treatment; $y$-axis: probability of the given treatment (or control); SUCRA = surface under the cumulative ranking curve). 
Azilsartan10mg (SMD $=-1.64,95 \% \mathrm{CI}-3.28$ to -0.01$)$, Losartan600mg vs. Azilsartan10mg (SMD =-6.89, 95\% CI -9.29 to -4.49$)$, Losartan30mg vs. Azilsartan1mg $(\mathrm{SMD}=-3.09,95 \% \mathrm{CI}-5.39$ to -0.79$)$, Losartan600mg vs. Azilsartan1mg (SMD $=-5.96,95 \% \mathrm{CI}-8.37$ to -3.55$)$, Losartan30mg vs. Azilsartan6mg (SMD $=-2.78,95 \%$ CI -5.09 to -0.47$)$, Losartan600mg vs. Azilsartan6mg $(\mathrm{SMD}=-5.64,95 \% \mathrm{CI}-8.07$ to -3.23$)$, Losartan30mg vs. Enalapril10mg (SMD $=-3.35,95 \% \mathrm{CI}-5.76$ to -0.94$)$, Losartan600mg vs. Enalapril110mg (SMD = -6.24, 95\% CI -8.73 to -3.70$)$, Losartan600mg vs. Enalapril12mg $(\mathrm{SMD}=-4.77,95 \% \mathrm{CI}-7.12$ to -2.42$)$, Olmesartan10mg vs. Enalapril12mg (SMD = 1.61, $95 \%$ CI 0.04 to 3.17), Telmisartan10mg vs. Enalapril12mg (SMD = 1.64, 95\% CI 0.08 to 3.21), Telmisartan5mg vs. Enalapril12mg $(\mathrm{SMD}=1.85,95 \%$ CI 0.23 to 3.47), Losartan600mg vs. Enalapril60mg (SMD = -5.04, $95 \%$ CI 7.51 to -2.57), Losartan 30mg vs. Losartan10mg (SMD=-2.95, $95 \%$ CI -5.26 to -0.63$)$, Losartan600mg vs. Losartan10mg $(\mathrm{SMD}=-5.81,95 \% \mathrm{CI}-8.24$ to -3.38$)$, and Losartan50mg vs. Losartan30mg (SMD = 2.38, $95 \%$ CI 0.97 to 4.67$)$.

Quantitative synthesis of the secondary outcomes Results of the pairwise meta-analysis of the secondary outcomes reported by at least 3 studies are shown in
Fig. 8. The Intervention groups reporting outcomes from different tissue sites in the study conducted by Moura et al. were included separately ("Moura2016a" and "Moura2016b"). Overall, 11 studies (Araujo et al., 2013a; Araujo et al., 2013b; Araújo et al., 2014; Li et al., 2019; Matos et al., 2013; Matos et al., 2014; Matos et al., 2015; Moura et al., 2016; Oliveira et al., 2019; Queiroz-Junior et al., 2015; Suda et al., 2013), 12 experimental intervention groups and 8 outcomes (IL-10, IL-1 $\beta$, TNF- $\alpha$, MDA, MPO, GSH, RANKL and number of TRAP+ cells/osteoclasts) were included in the pairwise meta-analysis. For the oxidative marker MDA, a significant difference was found for pooled Olmesartan (SMD $=-4.71,95 \% \mathrm{CI}-6.01$ to -3.41$)$ and Telmisartan (SMD $=-5.11,95 \% \mathrm{CI}-6.49$ to -3.73 ) doses, for Valsartan (SMD $=-5.20,95 \% \mathrm{CI}-8.39$ to - 2.01) and for the combined ARBs (SMD $=-4.92$, $95 \%$ CI -5.83 to -4.02 ). Only the pooled Telmisartan groups (SMD $=0.89,95 \% \mathrm{CI} 0.15$ to 1.63 ) and the combined ARBs (SMD $=0.62,95 \%$ CI 0.19 to 1.04 ) increased the antioxidative marker GSH. For MPO, all but the pooled Azilsartan groups resulted in a significantly lower MPO level leading to a significant overall effect (SMD $=-1.18,95 \% \mathrm{CI}-1.81$ to -0.54 ). Regarding IL-1 $\beta$, pooled Olmesartan and Telmisartan groups, respectively, were significantly different from
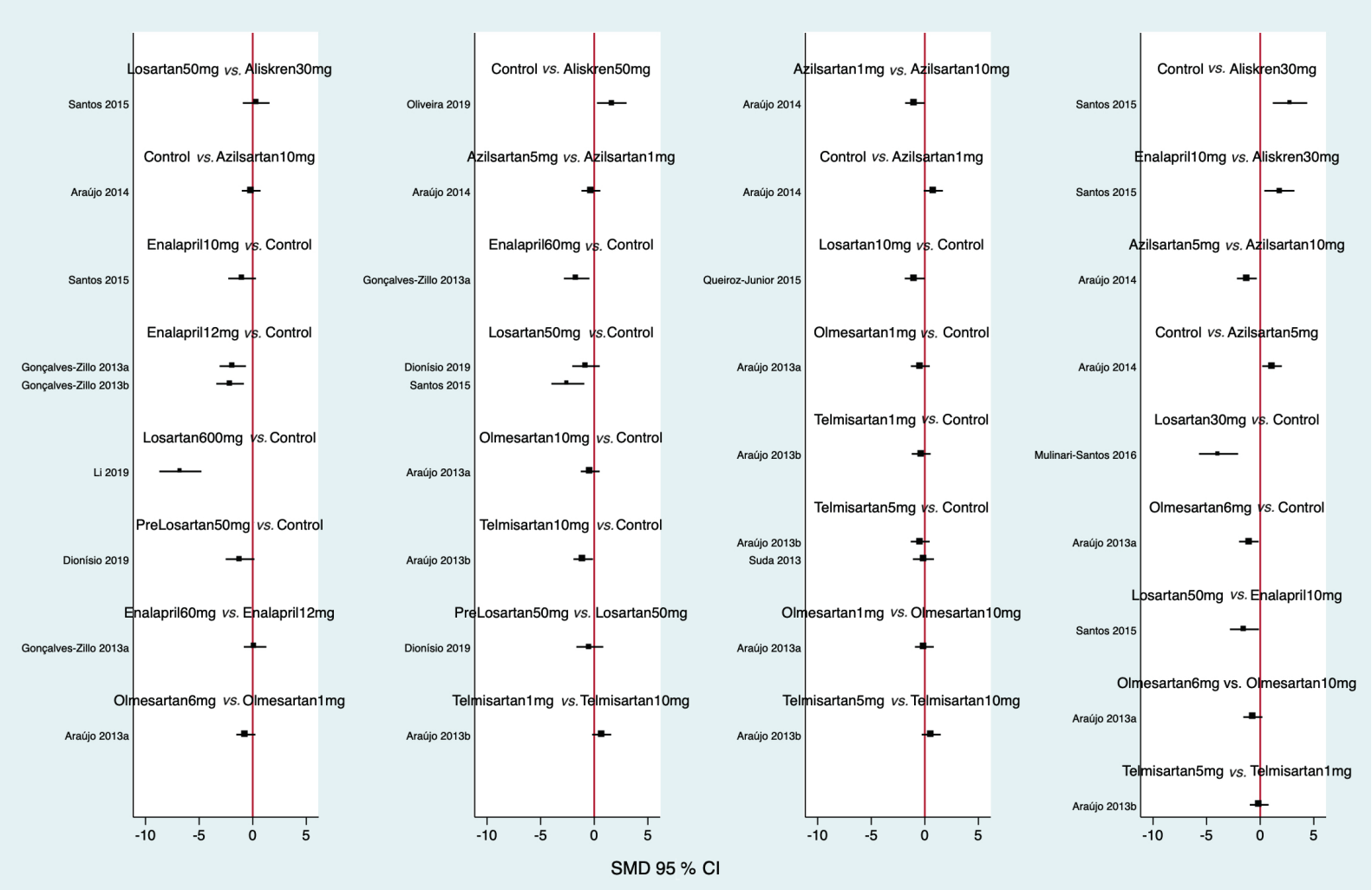

Fig. 7. Network forest plot of the network meta-analysis for the different doses of tRAS inhibitors and the comparator. The effect size (SMD) is shown with its $95 \%$ confidence interval. An SMD under 0 ("line of null effect") favours the intervention for the prevention of periodontal bone loss (How to read: A vs. B; A = Intervention, B = Comparator). Losartan600mg, Enalapril12mg, and Enalapril60mg are given as $\mathrm{mg} / \mathrm{L}$ in drinking water for the duration of the experiment. All other values are shown as $\mathrm{mg} / \mathrm{kg} / \mathrm{d}$ for the duration of the experiment. 

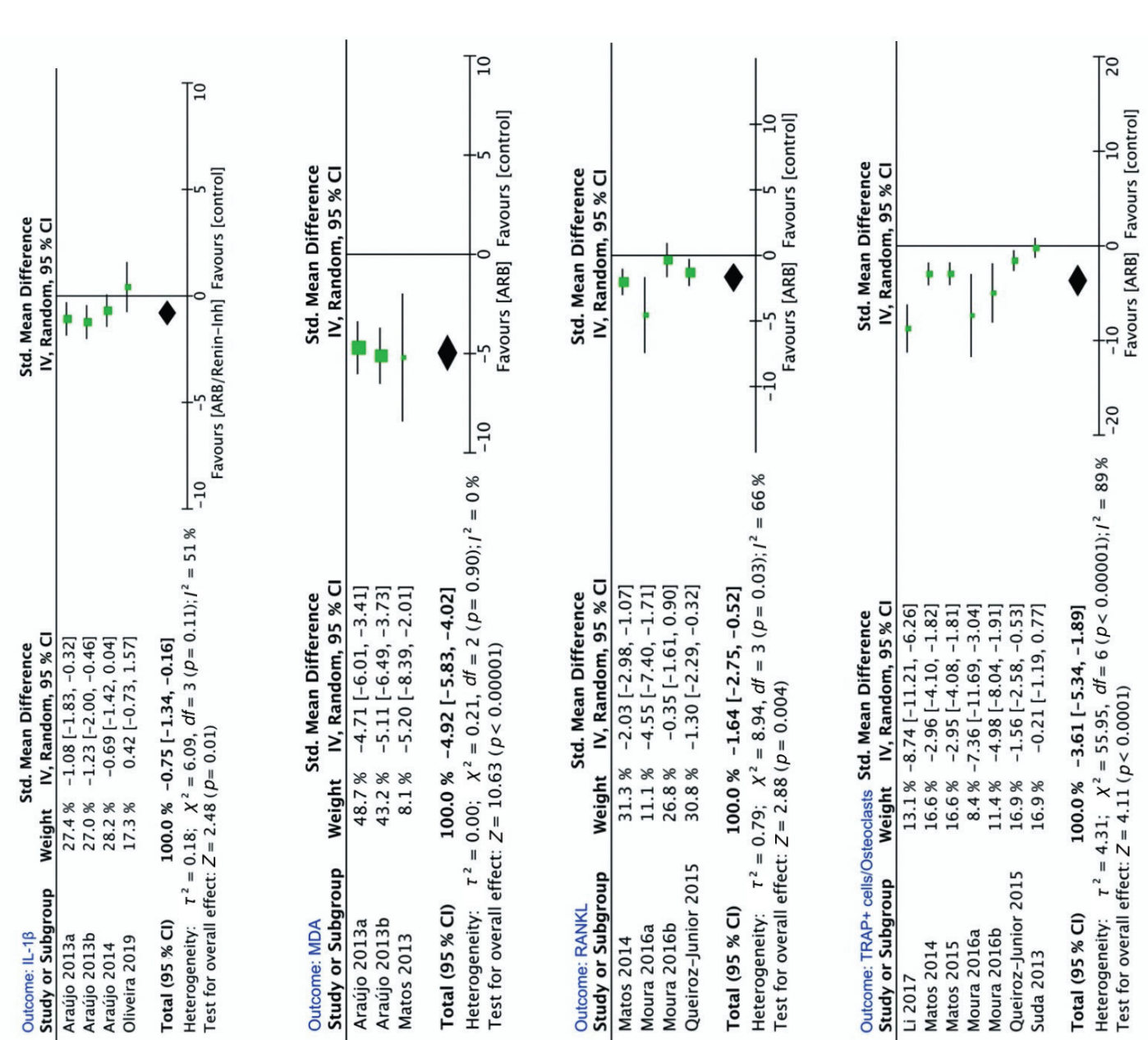

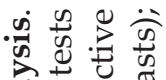
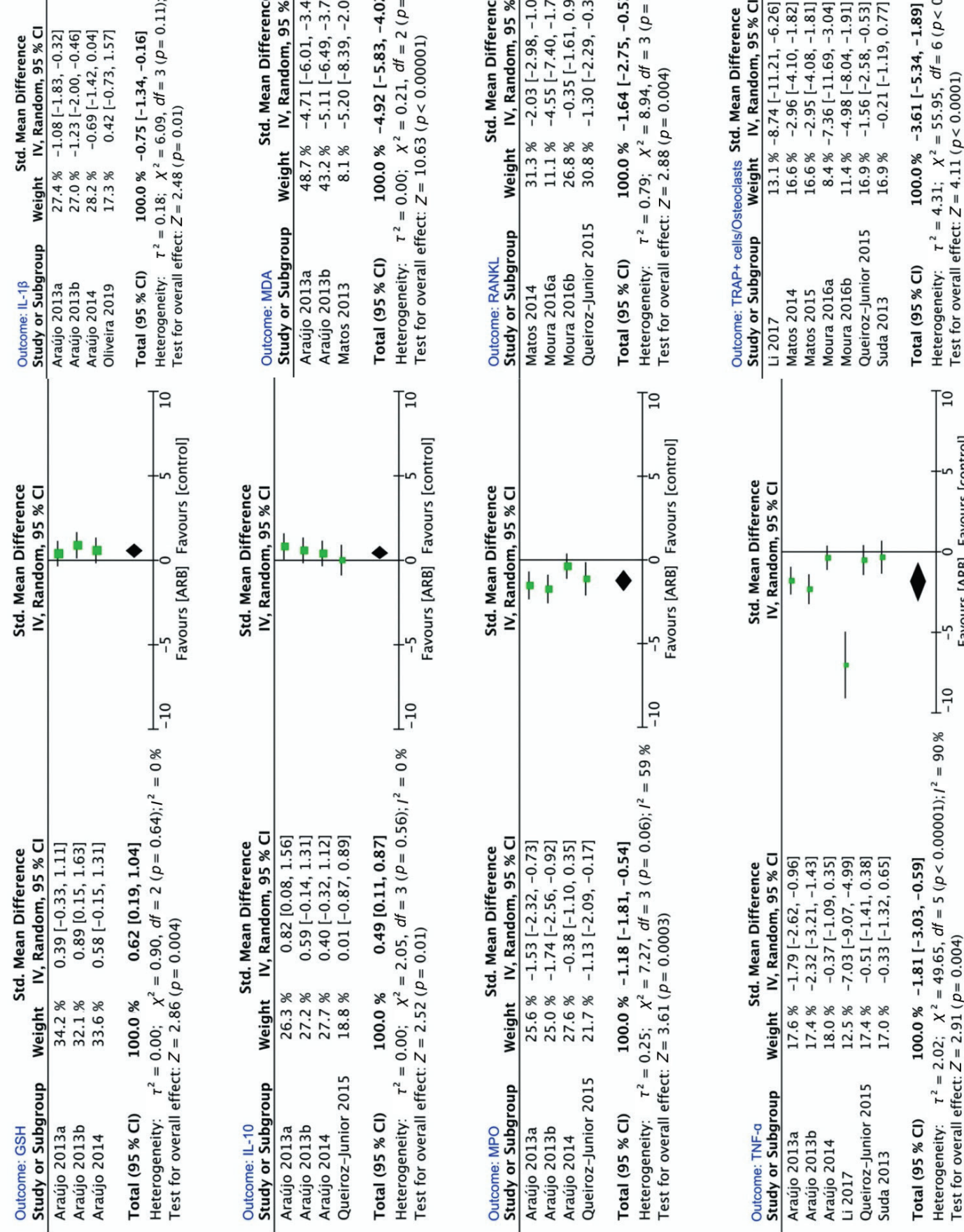

To 용

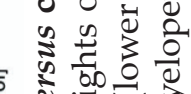
5 एँ

율

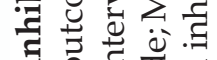

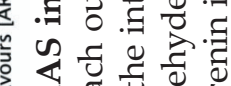

$\approx \approx$ o

논

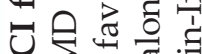

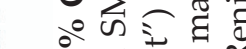

으 0 \&

ठ월

室至

क 50

돈

on $\approx$ :

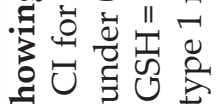

oㅇ $\dot{0}=$

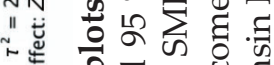

总

क्ष

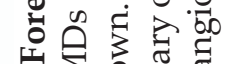

$\infty$ is o II

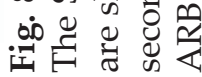


the control group. Further, the combined groups of tRAS inhibitors were significantly different from the control group $(\mathrm{SMD}=-0.75,95 \% \mathrm{CI}-1.34$ to -0.16$)$, but Aliskiren alone was not significantly different from the control group. For IL-10 only the pooled Olmesartan group and the combined ARBs reached statistical significance $(\mathrm{SMD}=0.49,95 \% \mathrm{CI} 0.11$ to 0.87 ). The Losartan group reported by Li et al. showed the strongest significant difference regarding TNF- $\alpha$ levels (SMD $=-7.03,95 \% \mathrm{CI}-9.07$ to -4.99 ). Additionally, pooled Olmesartan and Telmisartan groups showed significant differences compared to the control group, leading to a significant overall effect of the ARBs (SMD $=-1.81,95 \% \mathrm{CI}-3.03$ to - 0.59). All, but the Telmisartan group resulted in a significantly lower TRAP+ cell/osteoclast count compared to the untreated control group. The overall effect of the pooled ARBs was significantly different from that of the control group (SMD $=-3.61$, $95 \%$ CI - 5.34 to - 1.89). Finally, data for RANKL presented a significant difference for Valsartan $(10 \mathrm{mg} / \mathrm{kg} / \mathrm{d}$ for $7 \mathrm{~d})$, Losartan $(10 \mathrm{mg} / \mathrm{kg} / \mathrm{d}$ for $12 \mathrm{~h}$ ) and Losartan $(10 \mathrm{mg} / \mathrm{kg} / \mathrm{d}$ for $30 \mathrm{~d})$. Only one of the intervention groups ("compression site") reported by Moura et al. led to significantly lower RANKL levels. The combined effect showed statistical significance $(\mathrm{SMD}=-1.64,95 \% \mathrm{CI}-2.75$ to -0.52$)$. Significantly high between-study heterogeneity was found in the pairwise meta-analysis of the secondary outcomes TNF- $\alpha$ and TRAP + cells/osteoclasts. Heterogeneity was incorporated in a random-effects model as an analytical approach. Between reviewer explanations for the high heterogeneities in these 2 cases were the hypertension baseline characteristic in the study conducted by Li et al. (Li et al., 2019), for the outcome TNF- $\alpha$, and the experimental method of orthodontic force application in the study conducted by Moura et al. (Moura et al., 2016) for the outcome TRAP+cells/osteoclasts, respectively. When excluding these studies from the pairwise meta-analysis, heterogeneity was judged moderate with an $I^{2}<75 \%$.

\section{Risk of bias assessment}

The assessment of the risk of bias according to the items included in SYRCLE's tool is provided in Fig. 9. Overall, only a few experiments adequately reported the items included in SYRCLE's tool, leading to a high percentage of the judgment "unclear risk of bias". The best-reported items were "random group allocation (selection)" (8/17,47\%) and "group similar at baseline (selection)" (8/17, $47 \%)$. Santos et al. were reporting most of the Items (7/9) (Santos et al., 2015). The 5 studies conducted by Matos et al. were the studies reporting the fewest items and with the most positive judgments in the item category "other sources of bias" due to not reporting the sample sizes overall (Matos et al., 2016; Matos et al., 2019) and not adequately reporting sample sizes before the result section to evaluate drop-outs (Matos et al., 2013; Matos et al., 2014; Matos et al., 2015). The items

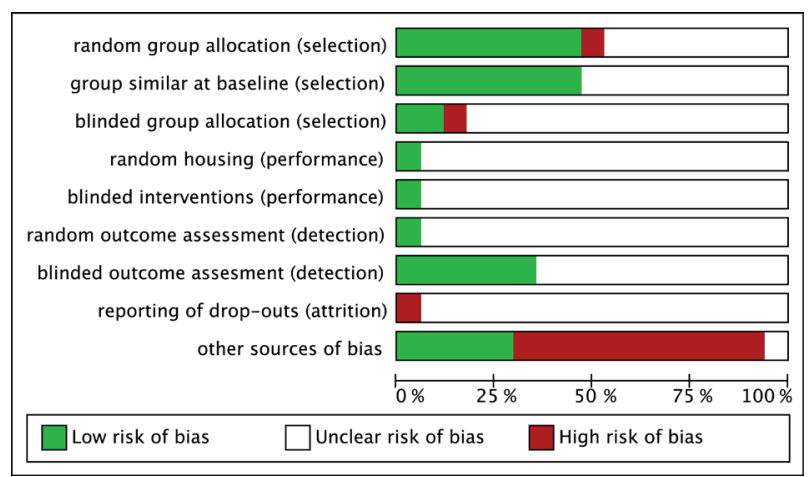

Fig. 9. Assessment of the risk of bias using the SYRCLE's tool.

"random housing (performance)" and "blinded interventions (performance)" were only reported by Santos et al. (Santos et al., 2015). Overall, the results of the assessment of bias are as expected for animal studies, which often lack adequate techniques to avoid the risk of bias or simply do not adequately report items to assess the risk of bias in animal studies.

\section{Discussion}

Severe periodontitis is still a global health problem leading to a huge socio-economic burden for the affected individuals and the healthcare system. New treatment approaches require intense and specified preclinical research and therefore this topic has been intensely researched in preclinical settings in recent years. There is a lack of information regarding the role of tRAS in the periodontal tissue. A summary of the evidence was required to pave the way for more specialised research and to transfer the findings into the clinical setting in humans. To the authors' best knowledge, this is the first systematic review evaluating the impact of periodontal tRAS inhibition on important surrogate and clinical endpoints in animal studies. We used a combination of pairwise- and network meta-analysis techniques to assess the outcomes after inhibition with different pharmacological intervention arms.

The data show, that tRAS inhibition with ARBs, renin-inhibitors, and ACE-inhibitors resulted in significantly lower periodontal bone loss when compared to the untreated experimental groups. We could not find a significant difference when comparing the different tRAS inhibitors with regards to bone loss in the network meta-analysis. Nevertheless, the treatment ranking suggests, that the renin-inhibitor Aliskiren was most effective in preventing periodontal bone loss. When comparing the different ARBs, the Losartan group performed significantly better than other ARBs. Furthermore, the group treated with $0.6 \mathrm{~g} / \mathrm{L}$ in drinking water for 8 weeks performed significantly better than all other tRAS inhibitor groups. Additionally, inhibition 
with tRAS inhibitors led to lower osteoclast counts, lower RANK and RANK-L levels, and higher OPG levels. Further, these findings for RANK, RANKL and OPG were present at $12 \mathrm{~h}$ of $\mathrm{tRAS}$ inhibition and were more pronounced after $30 \mathrm{~d}$ of $\mathrm{tRAS}$ inhibition. The oxidative markers were also affected by tRAS inhibition with ARBs, leading to a small increase in the antioxidative marker GSH, suggesting a lower oxidative stress level in the ARB treated groups, and a decrease of MPO and MDA levels - both markers which are usually increased during oxidative stress. From the surrogate markers related to inflammation, TNF- $\alpha$ was decreased in the pooled ARB group compared to the untreated control, and IL-1 $\beta$ was decreased in the pooled ARB and renin-inhibitor group compared to the untreated control. However, lower SMD was observed when excluding Aliskiren from the analysis, which alone showed no significant impact on IL-1 $\beta$ levels, thus this effect was only evident in the pooled ARB treated group. IL-10, an anti-inflammatory cytokine, seems to be higher in the combined ARB treated group; however, this finding failed to reach statistical significance.

Several limitations need to be addressed when interpreting these findings:

1. All studies included in this systematic review were conducted in rats and mice, thus translating the results to other animal species or humans should be performed carefully. Although rodent anatomy and physiology is not identical to that of humans, they are well established and reliable models for research on periodontal microbiology and immune response (Baker et al., 2000; Graves et al., 2008; Kantarci et al., 2015; Marchesan et al., 2018; Struillou et al., 2010). Moreover, several recent studies provided related findings for human gingival and periodontal tissues. For example, the presence of tRAS components in human gingival and periodontal ligament fibroblasts was recently confirmed (Monnouchi et al., 2011; Santos et al., 2015). Further, AT1R knockdown resulted in higher OPG levels (an inhibitor of bone resorption) and lower IL-1 $\beta$, IL-8 and IL-6 concentration (major inflammatory cytokines involved in periodontal diseases) in human gingival fibroblasts(Gabriele et al., 2017). Moreover, Angiotensin II was shown to induce prostaglandin E2 (which has an important role in the periodontal inflammatory process) release in human gingival fibroblasts, whereas the effect was inhibited by the AT1R antagonist FR-13,739 (Segawa et al., 2003).

2. Different methodological approaches were used by the authors to assess the impact of RAS-inhibition on primary and secondary outcomes, which limits the comparability. The use of ligatures is considered to not significantly induce inflammation and the effect is mainly dependent on accumulating bacteria. In comparison, direct injections of LPS and infection with periodontal pathogens have different mechanisms, such as inflammation triggering via toll-like receptors or modulation of the host subgingival biofilm (Marchesan et al., 2018; Struillou et al., 2010). Nevertheless, the effect measure was overall similarly distributed, as assessed in the random-effect paired meta-analysis. Interestingly, high heterogeneity was seen between the studies of Li et al. and Suda et al. for the outcome TNF- $\alpha$, although both used the periodontitis model based on an infection with Porphyromonas gingivalis. In contrast, the between-study heterogeneity, especially for the studies using ligature-induced periodontitis models and infection with Porphyromonas gingivalis can be considered comparable with the focus on the results of the heterogeneity test. The study conducted by Moura et al. showed a relatively high heterogeneity compared to the other studies for RANKL and the number of TRAP+ cells/osteoclasts. The orthodontic force application, which could affect bone remodelling processes in a different way from the other methodological examination methods could be one explanation.

3. Different tRAS inhibitors with different doses and experiment durations were compared and thus results should not be generalised for all tRAS inhibitors. It is important to know that within the group of ARBs there are also differences in the effective mechanism of the inhibitors. For example, it is stated that Losartan is not only blocking AT1R on the cell surface but also intracellular AT1Rs while Candesartan seems to remain surface-bound (Cook et al., 2001). Nevertheless, it is not recommendable for this systematic review to only focus on one specific tRAS inhibitor, as there is not enough evidence and it would possibly overlook other important relationships between tRAS inhibition and outcomes of interest. Furthermore, a subgroup or sensitivity analysis with consideration of duration, dose, and one specific tRAS inhibitor class was not possible with regards to the available evidence. With the network meta-analysis, the different intervention arms could be compared regardless of the methods used or duration of $\mathrm{tRAS}$ inhibition to present an overview, possible relationships for future comparison studies, and help to prevent duplicate reporting in prospective studies.

4. Multiple studies conducted by 2 research groups were included in this systematic review which may lead to bias and should be considered when interpreting the results. Especially, the studies conducted by the research group of Matos et al. were rated low with regards to the assessment of bias using the SYRCLE's tool. However, these studies were excluded from the quantitative syntheses, when no sample size was present. In this case, a qualitative synthesis was provided of the results and only included the significant findings. The other included studies can be considered as adequate with regards to the risk of bias in animal studies and are in accordance with the majority of animal studies, which often lack in reporting of important items for the assessment of the risk of bias ("unclear risk of bias") as the assessment of the risk of bias in systematic reviews of animal studies is far less 
common (Hooijmans et al., 2014; Sandercock and Roberts, 2002).

5. The exclusion criteria led to the exclusion of possibly important preclinical studies in human and animal cell cultures, which could also be of interest to the research topic and might lead to different results when analysed quantitatively. However, it would be not possible to include these cell culture studies in the network analysis as the presumption (similarity, transitivity, consistency) which should be confirmed a priori would not be satisfied. Thus, only a systematic literature review with a qualitative synthesis of the results would have been possible. Furthermore, as far as is known, there are no clinical studies in humans listed in the peer-reviewed literature evaluating RAS-inhibitors' impact on periodontal outcome parameters. Therefore, a systematic review focusing on human participants was not feasible. As a compromise, all available studies on this topic were considered in the discussion section, as part of the available evidence, regardless of the study type. Lastly, it must be mentioned that the task of this evidence-based systematic review was to summarise preclinical studies regarding the desired topic, to pave the way for more clinically oriented research. Definitive conclusions cannot be drawn regarding the clinical applicability in humans. Drug therapeutics mostly have side effects and limitations; and should therefore be considered to be an invasive therapeutical approach. Thus, intervention in this important host modulating system should be reserved for severe periodontitis and in cases where other non-invasive host modulating therapies (e.g. diet) fail.

To interpret the information retrieved in this systematic review properly, recent ex vivo studies regarding tRAS in the periodontal tissue and inflammation should also be considered. The study conducted by Choe et al. in 2019 evaluated the influence of Telmisartan on inflammatory mediators in murine macrophages stimulated with LPS from Prevotella intermedia, an important periodontitisassociated species (Choe et al., 2019). They found out, that Telmisartan led to significant inhibition of LPS-induced generation of inflammatory mediators, such as IL-1 $\beta$, which is in accordance with the presented data. In contrast, the study conducted by Gabriele et al. (2017) found an impairment of IL-1 $\beta$ induced secretion of proinflammatory mediators when silencing AT1R but not after Losartan treatment in human gingival fibroblasts and human periodontal fibroblast, suggesting a different control of inflammatory cytokines after AT1R knockdown and pharmacologic blockade by Losartan. One explanation for this finding could be the multiple regulatory mechanisms Losartan can affect. Ang II binding on AT1R is known to result in translocation of the Ang II/AT1R complex to the nuclear membrane and Losartan is known to block not only surface located AT1Rs, but also intracellular AT1Rs (Cook et al., 2001; Villar-Cheda et al., 2017). Further, the Losartan application for $14 \mathrm{~d}$ was shown to upregulate AT2R in the gingival tissue of rats with experimental induced periodontitis (Santos et al., 2015). This could promote the anti-inflammatory pathways of the cell via an alternative route (Dandona et al., 2007). Another explanation could be, that Telmisartan is also known to downregulate AT1R through the activation of peroxisome proliferatoractivated receptor-gamma (PPAR- $\gamma$ ), resulting in stronger inhibition of proinflammatory mechanisms (Imayama et al., 2006). In this case, Telmisartan, as a partial agonist of PPAR- $\gamma$, was the only ARB to show relevant activation of PPAR $-\gamma$ that can be achieved in plasma with conventional oral dosing (Benson et al., 2004).

Candesartan, as another widely used ARB, has been shown to inhibit LPS induced TLR4 upregulation (Dasu et al., 2009). AT1R and TLR4 seem to work synergistically and upregulation of TLR4 by Angiotensin 2 via AT1R has been stated in multiple studies (Biancardi et al., 2016; De Batista et al., 2014; Goel et al., 2018; Shirai et al., 2013; Wolf et al., 2006; Wu et al., 2009). An inhibition with Candesartan inhibited the TLR4/angiotensin II-induced NF-kB inflammatory pathway in a recent study (Goel et al., 2018). Moreover, LPS has been shown to increase the expression of AT1R (Li et al., 2015; Xianwei et al., 2012), whereas the LPS response was prevented with the ARB Candesartan (Sanchez-Lemus et al., 2008). There is further evidence, that AT1R in the periodontal tissue is upregulated (Gabriele et al., 2017; Nakamura et al., 2011) or more pronounced than AT2R (Santos et al., 2015) in the inflammation setting, whereas it is lower expressed in healthy periodontal tissue. In a recent study, bacteria-induced periodontitis resulted in a significant upregulation of Ang II concentrations and TLR4 mRNA, while the effect was inhibited with the ARB Losartan (Li et al., 2019). This finding was also found for Valsartan (Matos et al., 2014).

The expression of matrix metalloproteinases, such as MMP-1 and MMP-2, and the osteoclastogenic factor RANKL was shown to be correlated with the expression of the proinflammatory markers IL-1 $\beta$ and TNF- $\alpha$ in the course of experimental periodontal disease (Garlet et al., 2006). IL-1 $\beta$ upregulated RANKL, but not OPG and induced osteoclastogenesis in human cementoblasts (Huynh et al., 2017). An increase in the RANKL/OPG ratio is known to be one main feature of periodontitis (Belibasakis and Bostanci, 2012). The ARBs evaluated in this systematic review were shown to decrease RANKL, increase OPG, and thus resulting in a higher RANKL/OPG ratio and a decrease in the number of osteoclasts. However, it was not possible to evaluate the pathway that led to these results. It could be that the decrease in proinflammatory cytokines led to lower RANKL secretion, as stated above. Another hypothesis would be, that the ARBs directly inhibit the Ang II-mediated induction of RANKL expression in osteoblasts, leading to the lesser activation of osteoclasts, as described for Olmesartan (Shimizu et al., 2008). This 
would suggest that the AT1R pathway is directly involved in bone remodelling processes and might be an important therapeutic target as described recently by Zhao et al. in 2019 (Zhao et al., 2019).

\section{Conclusions}

Outcomes of this systematic review suggested an important role for the tRAS in periodontal tissue. The inhibition of its components in animal models led to a reduction of periodontal bone loss and reduced inflammation with different intensity, depending on the type of inhibitor used. Thus, the inhibition of tRAS components could be a new target approach for treating periodontal diseases in humans. Future research should, therefore, consider different mechanisms of tRAS inhibitors. However, some of the included studies were associated with certain limitations and a high risk of bias. More well-designed randomised preclinical and clinical studies are needed to adequately translate the present findings into clinical practice.

\section{Acknowledgments}

The article processing charge was funded by the Baden-Wuerttemberg Ministry of Science, Research and Art, and the University of Freiburg in the funding program Open Access Publishing.

\section{Author contributions}

BS, GL: Substantial contributions to study design, acquisition, analysis, interpretation of data, drafting the paper, revising it critically, and final approval. SÜ: Substantial contributions to acquisition of data, analysis, interpretation of data, revising the article critically, and final approval.

TB, VW, SP, ZL, and SG: Substantial contributions to study design, revising the article critically, and final approval.

MB: Substantial contributions to study design, interpretation of data, revising the article critically, and final approval.

JPW: Substantial contributions to study design, interpretation of data, drafting the article, revising it critically, and final approval.

BS and GL take responsibility for the integrity of the work as a whole, from inception to the finished article.

\section{Role of the funding source}

Gernot Lang was supported by the Berta-OttensteinProgramme for Advanced Clinician Scientists, Faculty of Medicine, University of Freiburg, Germany.

\section{Conflict of interest}

The authors of the present manuscript declare that they have nothing to disclose.

\section{References}

Abadir PM, Foster DB, Crow M, Cooke CA, Rucker JJ, Jain A, Smith BJ, Burks TN, Cohn RD, Fedarko NS, Carey RM, O'Rourke B, Walston JD (2011) Identification and characterization of a functional mitochondrial angiotensin system. Proc Natl Acad Sci U S A 108: 14849-14854.

Altman DG, Bland JM (2005) Standard deviations and standard errors. BMJ 331: 903.

Alzayadneh EM, Chappell MC (2015) Nuclear expression of renin-angiotensin system components in NRK-52E renal epithelial cells. J Renin Angiotensin Aldosterone Syst 16: 1135-1148.

Araujo AA, Souza TO, Moura LM, Brito GAC, Aragao KS, Araujo LS, Medeiros CAX, Alves MSCF, Araujo RFJ (2013a) Effect of telmisartan on levels of IL-1, TNF- $\alpha$, down-regulated COX-2, MMP-2, MMP-9 and RANKL/RANK in an experimental periodontitis model. J Clin Periodontol 40: 1104-1111.

Araújo AA de, Varela H, Brito GA de C, Medeiros CACX de, Araújo L de S, do Nascimento JHO, de Araújo Júnior RF (2014) Azilsartan increases levels of IL-10, down-regulates MMP-2, MMP-9, RANKL/ RANK, Cathepsin $\mathrm{K}$ and up-regulates OPG in an experimental periodontitis model. PLoS ONE 9: e96750. DOI: 10.1371/journal.pone.0096750.

Araujo AA, Lopes de Souza G, Souza TO, de Castro Brito GA, Saboia Aragao K, Xavier de Medeiros CA, Lourenco Y, do Socorro Costa Feitosa Alves M, Fernandes de Araujo RJ (2013b) Olmesartan decreases IL-1 $\beta$ and TNF- $\alpha$ levels; downregulates MMP-2, MMP-9, COX-2, and RANKL; and upregulates OPG in experimental periodontitis. Naunyn Schmiedebergs Arch Pharmacol 386: 875-884.

Baker PJ, Dixon M, Roopenian DC (2000) Genetic control of susceptibility to Porphyromonas gingivalisinduced alveolar bone loss in mice. Infect Immun 68: 5864-5868.

Belibasakis GN, Bostanci N (2012) The RANKLOPG system in clinical periodontology. J Clin Periodontol. 39: 239-248.

Benson SC, Pershadsingh HA, Ho CI, Chittiboyina A, Desai P, Pravenec M, Qi N, Wang J, Avery MA, Kurtz TW (2004) Identification of Telmisartan as a unique angiotensin II receptor antagonist with selective PPAR $\gamma$-modulating activity. Hypertension 43: 993-1002.

Berggreen E, Heyeraas KJ (2003) Role of K+ATP channels, endothelin A receptors, and effect of angiotensin II on blood flow in oral tissues. J Dent Res 82: 33-37.

Biancardi VC, Bomfim GF, Reis WL, Al-Gassimi S, Nunes KP (2017) The interplay between angiotensin II, TLR4 and hypertension. Pharmacological Research 120: $88-96$

Biancardi VC, Stranahan AM, Krause EG, de Kloet AD, Stern JE (2016) Cross talk between AT1 receptors and Toll-like receptor 4 in microglia contributes to angiotensin II-derived ROS production in the 
hypothalamic paraventricular nucleus. Am J Physiol Heart Circ Physiol 310: H404-415.

Chabrashvili T, Kitiyakara C, Blau J, Karber A, Aslam S, Welch WJ, Wilcox CS (2003) Effects of ANG II type 1 and 2 receptors on oxidative stress, renal NADPH oxidase, and SOD expression. Am J Physiol Regul Integr Comp Physiol 285: R117-R124.

Chaimani A, Higgins JPT, Mavridis D, Spyridonos P, Salanti G (2013) Graphical tools for network metaanalysis in STATA. PLoS ONE 8: e76654. DOI: 10.1371/ journal.pone.0076654.

Choe S-H, Choi E-Y, Hyeon J-Y, Keum BR, Choi IS, Kim S-J (2019) Telmisartan, an angiotensin II receptor blocker, attenuates Prevotella intermedia lipopolysaccharide-induced production of nitric oxide and interleukin-1 $\beta$ in murine macrophages. Int Immunopharmacol 75: 105750.

Cipriani A, Higgins JPT, Geddes JR, Salanti G (2013) Conceptual and technical challenges in network meta-analysis. Ann Intern Med 159: 130-137.

Cohen J (1960) A coefficient of agreement for nominal scales. Educ Psychol Meas 20: 37-46.

Cook JL, Zhang Z, Re RN (2001) In vitro evidence for an intracellular site of angiotensin action. Circ Res 89: 1138-1146.

Dandona P, Dhindsa S, Ghanim H, Chaudhuri A (2007) Angiotensin II and inflammation: the effect of angiotensin-converting enzyme inhibition and angiotensin II receptor blockade. J Hum Hypertens 21: 20-27.

Darveau RP (2010) Periodontitis: a polymicrobial disruption of host homeostasis. Nat Rev Microbiol 8: 481-490.

Dasu MR, Riosvelasco AC, Jialal I (2009) Candesartan inhibits Toll-like receptor expression and activity both in vitro and in vivo. Atherosclerosis 202: 76-83.

De Batista PR, Palacios R, Martín A, Hernanz R, Médici CT, Silva MASC, Rossi EM, Aguado A, Vassallo DV, Salaices M, Alonso MJ (2014) Toll-like receptor 4 upregulation by angiotensin II contributes to hypertension and vascular dysfunction through reactive oxygen species production. PLoS ONE 9: e104020. DOI: 10.1371/journal.pone.0104020.

Dionisio TJ, Souza GP, Colombini-Ishikiriama BL, Garbieri TF, Parisi VA, Oliveira GM, Cano IP, Rodini CO, Oliveira SHP, Greene AS, Santos CF (2019) AT1 receptor antagonism promotes bone loss attenuation in experimental periodontitis, block inflammatory mediators, upregulate antioxidant enzymes and bone formation markers. J Periodontol 91: 533-544.

Dye BA (2012) Global periodontal disease epidemiology: Global periodontal disease epidemiology. Periodontol 2000 58: 10-25.

Dzau VJ, Re R (1994) Tissue angiotensin system in cardiovascular medicine. A paradigm shift? Circulation 89: 493-498.

Filipeanu CM, Henning RH, Nelemans SA, de Zeeuw D (2001) Intracellular angiotensin II: from myth to reality? J Renin Angiotensin Aldosterone Syst 2: 219-226.
Frencken JE, Sharma P, Stenhouse L, Green D, Laverty D, Dietrich T (2017) Global epidemiology of dental caries and severe periodontitis - a comprehensive review. J Clin Periodontol 44 Suppl 18: S94-S105.

Gabriele LG, Morandini AC, Dionísio TJ, Santos CF (2017) Angiotensin II type 1 receptor knockdown impairs interleukin-1 $\beta$-induced cytokines in human periodontal fibroblasts. J Periodontol 88: e1-e11.

Garlet GP, Cardoso CR, Silva TA, Ferreira BR, Avila-Campos MJ, Cunha FQ, Silva JS (2006) Cytokine pattern determines the progression of experimental periodontal disease induced by Actinobacillus actinomycetemcomitans through the modulation of MMPs, RANKL, and their physiological inhibitors. Oral Microbiol Immunol 21: 12-20.

Gebre AK, Altaye BM, Atey TM, Tuem KB, Berhe DF (2018) Targeting renin-angiotensin system against Alzheimer's disease. Front Pharmacol 9: 440.

Gebru Y, Diao T-Y, Pan H, Mukwaya E, Zhang Y (2013) Potential of RAS inhibition to improve metabolic bone disorders. Biomed Res Int 2013: 932691.

Goel R, Bhat SA, Hanif K, Nath C, Shukla R (2018) Angiotensin II receptor blockers attenuate lipopolysaccharide-induced memory impairment by modulation of NF- $\kappa \mathrm{B}$-mediated BDNF/CREB expression and apoptosis in spontaneously hypertensive rats. Mol Neurobiol 55: 1725-1739.

Goncalves-Zillo TO, Pugliese LS, Sales VMT, Mori MA da S, Squaiella-Baptistao CC, Longo-Maugeri IM, Lopes JD, de Oliveira SM, Monteiro AC, Pesquero JB (2013) Increased bone loss and amount of osteoclasts in kinin B1 receptor knockout mice. J Clin Periodontol 40: 653-660.

Graves DT, Fine D, Teng Y-TA, Van Dyke TE, Hajishengallis G (2008) The use of rodent models to investigate host-bacteria interactions related to periodontal diseases. J Clin Periodontol 35: 89-105.

Graves DT, Cochran D (2003) The contribution of interleukin-1 and tumor necrosis factor to periodontal tissue destruction. J Periodontol 74: 391-401.

Greenwell H (2001) Position paper: guidelines for periodontal therapy. J Periodontol 72: 1624-1628.

Gwathmey TM, Alzayadneh EM, Pendergrass KD, Chappell MC (2012) Novel roles of nuclear angiotensin receptors and signaling mechanisms. Am J Physiol Regul Integr Comp Physiol 302: R518-R530.

Higgins JPT, Thompson SG, Deeks JJ, Altman DG (2003) Measuring inconsistency in meta-analyses. BMJ 327: 557-560.

Hooijmans CR, Rovers MM, de Vries RBM, Leenaars M, Ritskes-Hoitinga M, Langendam MW (2014) SYRCLE's risk of bias tool for animal studies. BMC Med Res Methodol 14: 43.

Huynh NC-N, Everts V, Pavasant P, Ampornaramveth RS (2017) Interleukin-1 $\beta$ induces human cementoblasts to support osteoclastogenesis. Int J Oral Sci 9: e5. DOI: 10.1038/ijos.2017.45.

Imayama I, Ichiki $\mathrm{T}$, Inanaga $\mathrm{K}$, Ohtsubo $\mathrm{H}$, Fukuyama K, Ono H, Hashiguchi Y, Sunagawa K 
(2006) Telmisartan downregulates angiotensin II type 1 receptor through activation of peroxisome proliferator-activated receptor $\gamma$. Cardiovasc Res 72: 184-190.

Kantarci A, Hasturk H, Van Dyke TE (2006) Hostmediated resolution of inflammation in periodontal diseases. Periodontol 2000 40: 144-163.

Kantarci A, Hasturk H, Van Dyke TE (2015) Animal models for periodontal regeneration and peri-implant responses. Periodontol 2000 68: 66-82.

Karlsson C, Lindell K, Ottosson M, Sjöström L, Carlsson B, Carlsson LM (1998) Human adipose tissue expresses angiotensinogen and enzymes required for its conversion to angiotensin II. J Clin Endocrinol Metab 83: 3925-3929.

Kassebaum NJ, Bernabé E, Dahiya M, Bhandari B, Murray CJL, Marcenes W (2014) Global burden of severe periodontitis in 1990-2010: a systematic review and meta-regression. J Dent Res 93: 1045-1053.

van Kats JP, Danser AH, van Meegen JR, Sassen LM, Verdouw PD, Schalekamp MA (1998) Angiotensin production by the heart: a quantitative study in pigs with the use of radiolabeled angiotensin infusions. Circulation 98: 73-81.

Krayer JW, Leite RS, Kirkwood KL (2010) Nonsurgical chemotherapeutic treatment strategies for the management of periodontal diseases. Dent Clin North Am 54: 13-33.

Li H-P, Qiu H-B, Wang H-Q (2015) Effect of lipopolysaccharide on angiotensin II type 1 receptor expression and function in human pulmonary microvascular endothelial cells. Mol Med Rep 12: 8289-8293.

Li J, Xiao X, Wei W, Ding H, Yue Y, Tian Y, Nabar NR, Liu Z, Yang Z, Wang M (2019) Inhibition of angiotensin II receptor I prevents inflammation and bone loss in periodontitis. J Periodontol 90: 208-216.

Lindpaintner K, Ganten D (1991) The cardiac renin-angiotensin system. An appraisal of present experimental and clinical evidence. Circ Res 68: 905921.

Lu H, Boustany-Kari CM, Daugherty A, Cassis LA (2007) Angiotensin II increases adipose angiotensinogen expression. Am J Physiol Endocrinol Metab 292: E1280-1287.

Lundergan WP, Ferry D, Kobayashi H, Snowdowne KW (1999) Angiotensin-II increases cytoplasmic calcium, cell number and total DNA for human periodontal ligamental cells in vitro. J Periodontal Res 34: 223-228.

Lv J, Chen Q, Shao Y, Chen Y, Shi J (2015) Crosstalk between angiotensin-II and toll-like receptor 4 triggers a synergetic inflammatory response in rat mesangial cells under high glucose conditions. Biochem Biophys Res Commun 459: 264-269.

Lynn H, Kwok T, Wong SYS, Woo J, Leung PC (2006) Angiotensin converting enzyme inhibitor use is associated with higher bone mineral density in elderly Chinese. Bone 38: 584-588.

Marcenes W, Kassebaum NJ, Bernabé E, Flaxman A, Naghavi M, Lopez A, Murray CJL (2013) Global burden of oral conditions in 1990-2010: a systematic analysis. J Dent Res 92: 592-597.

Marchesan J, Girnary MS, Jing L, Miao MZ, Zhang S, Sun L, Morelli T, Schoenfisch MH, Inohara N, Offenbacher S, Jiao Y (2018) An experimental murine model to study periodontitis. Nat Protoc 13: $2247-$ 2267.

Martin-Cabezas R, Seelam N, Petit C, Agossa K, Gaertner S, Tenenbaum H, Davideau J-L, Huck O (2016) Association between periodontitis and arterial hypertension: a systematic review and meta-analysis. Am Heart J 180: 98-112.

Matos G, Perdomo L, Alvarez M, Anita I, Garrido D (2015) Papel del receptor AT1 de la angiotensina II en la remodelación ósea que ocurre durante la periodontitis experimental en la rata. Rev Fac Farm Univ Cent Venez 78: 84-93.

Matos M, Anita I, Billet E, Garrido M (2016) Citocinas pro-inflamatorias en la enfermedad periodontal experimental: Efecto del valsartán. Rev Fac Farm Univ Cent Venez 79: 17-27.

Matos M, Anita I, Billet E, Garrido M (2019) Efecto del valsartán sobre los niveles de citocinas y quimiocinas salivales en la enfermedad periodontal experimental. Rev Fac Farm Univ Cent Venez 82: 27-45.

Matos M, Billet E, Mathison Y, Anita I (2013) Generación de especies reactivas de oxígeno en la periodontitis experimental en la rata. Papel del receptor AT1 y la NAD(P)H oxidasa. Rev Fac Farm Univ Cent Venez 76: 58-66.

Matos M, Perdomo L, Alvarez M, Anita I, Garrido M (2014) El valsartán previene la resorción ósea en la periodontitis experimental. Periodonciay Osteointegración 24: 289-295.

Miggin SM (2006) New insights into the regulation of TLR signaling. J Leukoc Biol 80: 220-226.

Monnouchi S, Maeda H, Fujii S, Tomokiyo A, Kono K, Akamine A (2011) The roles of angiotensin II in stretched periodontal ligament cells. J Dent Res 90: 181-185.

Montecucco F, Pende A, Mach F (2009) The renin-angiotensin system modulates inflammatory processes in atherosclerosis: evidence from basic research and clinical studies. Mediators Inflamm 2009: 1-13.

Morimoto R, Akeda K, Iida R, Nishimura A, Tsujii M, Obata S, Kasai Y, Uchida A, Sudo A (2013) Tissue renin-angiotensin system in the intervertebral disc. Spine (Phila Pa 1976) 38: E129-E136.

Moulik S, Speth RC, Turner BB, Rowe BP (2002) Angiotensin II receptor subtype distribution in the rabbit brain. Exp Brain Res 142: 275-283.

Moura AP, Montalvany-Antonucci CC, Taddei SR de A, Queiroz-Junior CM, Biguetti CC, Garlet GP, Ferreira AJ, Teixeira MM, Silva TA, Andrade IJ (2016) Effects of angiotensin II type I receptor blocker losartan on orthodontic tooth movement. Am J Orthod Dentofacial Orthop 149: 358-365.

Mulinari-Santos G, Santos JSD, Palin LP, Silva ACE da, Antoniali C, Faverani LP, Okamoto R 
(2019) Losartan improves alveolar bone dynamics in normotensive rats but not in hypertensive rats. J Appl Oral Sci 27: e20180574.

Nadlonek N, Lee JH, Reece TB, Weyant MJ, Cleveland JC, Meng X, Fullerton DA (2013) Interleukin-1 beta induces an inflammatory phenotype in human aortic valve interstitial cells through nuclear factor kappa beta. Ann Thorac Surg 96: 155-162.

Nakagami H, Morishita R (2009) [Hormones and osteoporosis update. Effect of angiotensin II on bone metabolism]. Clin Calcium 19: 997-1002.

Nakamura T, Hasegawa-Nakamura K, Sakoda K, Matsuyama T, Noguchi K (2011) Involvement of angiotensin II type 1 receptors in interleukin-1 $\beta$ induced interleukin-6 production in human gingival fibroblasts. Eur J Oral Sci 119: 345-351.

Ohuchi N, Hayashi K, Koike K, Kizawa Y, Kusama T, Ohsawa M, Taniguchi Y, Iwamoto K, Sano M, Murakami H (2004) Pharmacological properties of angiotensin II receptors in cultured rabbit gingival fibroblasts. Comp Biochem Physiol C Toxicol Pharmacol 137: 281-289.

Ohuchi N, Koike K, Sano M, Kusama T, Kizawa Y, Hayashi K, Taniguchi Y, Ohsawa M, Iwamoto K, Murakami H (2002) Proliferative effects of angiotensin II and endothelin-1 on guinea pig gingival fibroblast cells in culture. Comp Biochem Physiol C Toxicol Pharmacol 132: 451-460.

Oliveira SHP, Brito VGB, Frasnelli SCT, Ribeiro B da S, Ferreira MN, Queiroz DP, Beltan CT, Lara VS, Santos CF (2019) Aliskiren attenuates the inflammatory response and wound healing process in diabetic mice with periodontal disease. Front Pharmacol 10: 708.

Paul M, Bachmann J, Ganten D (1992) The tissue renin-angiotensin systems in cardiovascular disease. Trends Cardiovasc Med 2: 94-99.

Petersen PE, Ogawa H (2012) The global burden of periodontal disease: towards integration with chronic disease prevention and control: global periodontal health. Periodontol 2000 60: 15-39.

Pihlstrom BL, Michalowicz BS, Johnson NW (2005) Periodontal diseases. Lancet 366: 1809-1820.

Preshaw PM (2018) Host modulation therapy with anti-inflammatory agents. Periodontol 2000 76: 131-149.

Queiroz-Junior CM, Silveira KD, de Oliveira CR, Moura AP, Madeira MFM, Soriani FM, Ferreira AJ, Fukada SY, Teixeira MM, Souza DG, da Silva TA (2015) Protective effects of the angiotensin type 1 receptor antagonist losartan in infection-induced and arthritis-associated alveolar bone loss. J Periodontal Res 50: 814-823.

Ramalingam L, Menikdiwela K, LeMieux M, Dufour JM, Kaur G, Kalupahana N, MoustaidMoussa N (2017) The renin angiotensin system, oxidative stress and mitochondrial function in obesity and insulin resistance. Biochim Biophys Acta Mol Basis Dis 1863: 1106-1114.
Re RN (2018) Role of intracellular angiotensin II. Am J Physiol Heart Circ Physiol 314: H766-H771.

Reddy MS, Geurs NC, Gunsolley JC (2003) Periodontal host modulation with antiproteinase, anti-inflammatory, and bone-sparing agents. a systematic review. Ann Periodontol 8: 12-37.

Remuzzi G, Perico N, Macia M, Ruggenenti P (2005) The role of renin-angiotensin-aldosterone system in the progression of chronic kidney disease. Kidney Int Suppl: S57-S65.

Ruiz-Ortega M, Lorenzo O, Rupérez M, Esteban V, Suzuki Y, Mezzano S, Plaza JJ, Egido J (2001) Role of the renin-angiotensin system in vascular diseases: expanding the field. Hypertension 38: 1382-1387.

Salanti G, Ades AE, Ioannidis JPA (2011) Graphical methods and numerical summaries for presenting results from multiple-treatment meta-analysis: an overview and tutorial. J Clin Epidemiol 64: 163-171.

Sanchez-Lemus E, Murakami Y, Larrayoz-Roldan IM, Moughamian AJ, Pavel J, Nishioku T, Saavedra JM (2008) Angiotensin II AT receptor blockade decreases lipopolysaccharide-induced inflammation in the rat adrenal gland. Endocrinology 149: 51775188.

Sandercock P, Roberts I (2002) Systematic reviews of animal experiments. Lancet 360: 586.

Santos CF, Morandini AC, Dionisio TJ, Faria FA, Lima MC, Figueiredo CM, Colombini-Ishikiriama BL, Sipert CR, Maciel RP, Akashi AP, Souza GP, Garlet GP, Rodini CO, Amaral SL, Becari C, Salgado MC, Oliveira EB, Matus I, Didier DN, Greene AS (2015) Functional local renin-angiotensin system in human and rat periodontal tissue. PLoS ONE 10: e0134601. DOI: 10.1371/journal.pone.0134601.

Santos CF, Akashi AE, Dionísio TJ, Sipert CR, Didier DN, Greene AS, Oliveira SHP, Pereira HJV, Becari C, Oliveira EB, Salgado MCO (2009) Characterization of a local renin-angiotensin system in rat gingival tissue. J Periodontol 80: 130-139.

Segawa M, Nakao S, Ogata Y, Sugiya H, Furuyama S (2003) Angiotensin II induces prostaglandin E(2) release in human gingival fibroblasts. Life Sci 72: 795-803.

Shimizu H, Nakagami H, Osako MK, Hanayama R, Kunugiza Y, Kizawa T, Tomita T, Yoshikawa H, Ogihara T, Morishita R (2008) Angiotensin II accelerates osteoporosis by activating osteoclasts. FASEB J 22: 2465-2475.

Shirai Y, Yoshiji H, Noguchi R, Kaji K, Aihara Y, Douhara A, Moriya K, Namisaki T, Kawaratani H, Fukui H (2013) Cross talk between toll-like receptor-4 signaling and angiotensin-II in liver fibrosis development in the rat model of non-alcoholic steatohepatitis: Innate immunity in the liver fibrosis. J Gastroenterol Hepatol 28: 723-730.

Solomon DH, Mogun H, Garneau K, Fischer MA (2011) Risk of fractures in older adults using antihypertensive medications. J Bone Miner Res 26: 1561-1567. 
Struillou X, Boutigny H, Soueidan A, Layrolle P (2010) Experimental animal models in periodontology: a review. Open Dent J 4: 37-47.

Suda N, Moriyama K, Ganburged G (2013) Effect of angiotensin II receptor blocker on experimental periodontitis in a mouse model of Marfan syndrome. Infect Immun 81: 182-188.

Tigerstedt R, Bergman PQ (1898) Niere und Kreislauf ${ }^{1}$. Skandinavisches Archiv Für Physiologie 8: 223-271.

Tonetti MS, Jepsen S, Jin L, Otomo-Corgel J (2017) Impact of the global burden of periodontal diseases on health, nutrition and wellbeing of mankind: A call for global action. J Clin Periodontol 44: 456-462.

Tricco AC, Lillie E, Zarin W, O'Brien KK, Colquhoun H, Levac D, Moher D, Peters MDJ, Horsley T, Weeks L, Hempel S, Akl EA, Chang C, McGowan J, Stewart L, Hartling L, Aldcroft A, Wilson MG, Garritty C, Lewin S, Godfrey CM, Macdonald MT, Langlois EV, Soares-Weiser K, Moriarty J, Clifford T, Tunçalp Ö, Straus SE (2018) PRISMA extension for scoping reviews (PRISMA-ScR): checklist and explanation. Ann Intern Med 169: 467-473.

Valenzuela R, Costa-Besada MA, IglesiasGonzalez J, Perez-Costas E, Villar-Cheda B, GarridoGil P, Melendez-Ferro M, Soto-Otero R, Lanciego JL, Henrion D, Franco R, Labandeira-Garcia JL (2016) Mitochondrial angiotensin receptors in dopaminergic neurons. Role in cell protection and aging-related vulnerability to neurodegeneration. Cell Death Dis 7: e2427-e2427.

Viafara-Garcia SM, Morantes SJ, Chacon-Quintero Y, Castillo DM, Lafaurie GI, Buitrago DM (2019) Repeated Porphyromonas gingivalis W83 exposure leads to release pro-inflammatory cytokynes and angiotensin II in coronary artery endothelial cells. Sci Rep 9: 19379.

Villar-Cheda B, Costa-Besada MA, Valenzuela R, Perez-Costas E, Melendez-Ferro M, LabandeiraGarcia JL (2017) The intracellular angiotensin system buffers deleterious effects of the extracellular paracrine system. Cell Death Dis 8: e3044-e3044.

de Vries RBM, Hooijmans CR, Langendam MW, van Luijk J, Leenaars M, Ritskes-Hoitinga $M$, Wever KE (2015) A protocol format for the preparation, registration and publication of systematic reviews of animal intervention studies: protocol format for animal systematic reviews. Evid Based Med 2: e00007.

White IR (2015) Network meta-analysis. Stata J 15: 951-985.

White IR, Barrett JK, Jackson D, Higgins JPT (2012) Consistency and inconsistency in network meta-analysis: model estimation using multivariate meta-regression. Res Synth Methods 3: 111-125.

Woelber JP, Tennert C (2020) Chapter 13: Diet and periodontal diseases. Monogr Oral Sci 28: 125-133.

Wolf G, Bohlender J, Bondeva T, Roger T, Thaiss F, Wenzel UO (2006) Angiotensin II upregulates toll- like receptor 4 on mesangial cells. J Am Soc Nephrol 17: 1585-1593.

Wu J, Yang $X$, Zhang Y-F, Zhou S-F, Zhang R, Dong X-Q, Fan J-J, Liu M, Yu X-Q (2009) Angiotensin II upregulates Toll-like receptor 4 and enhances lipopolysaccharide-induced CD40 expression in rat peritoneal mesothelial cells. Inflamm Res 58: 473-482.

Wu Z-H (2006) Molecular linkage between the kinase ATM and NF- B signaling in response to genotoxic stimuli. Science 311: 1141-1146.

Xianwei W, Magomed K, Ding Z, Sona M, Jingjun L, Shijie L, Mehta JL (2012) Cross-talk between inflammation and angiotensin II: Studies based on direct transfection of cardiomyocytes with AT1R and AT2R cDNA. Exp Biol Med (Maywood) 237: 13941401.

Zhang Y, Diao T-Y, Gu S-S, Wu S-Y, Gebru YA, Chen X, Wang J-Y, Ran S, Wong M-S (2014) Effects of angiotensin II type 1 receptor blocker on bones in mice with type 1 diabetes induced by streptozotocin. J Renin Angiotensin Aldosterone Syst 15: 218-227.

Zhao J, Yang H, Chen B, Zhang R (2019) The skeletal renin-angiotensin system: a potential therapeutic target for the treatment of osteoarticular diseases. Int Immunopharmacol 72: 258-263.

\section{Web references}

1. Self-programmed Stata routines http://www. mtm.uoi.gr [5/11/20].

2. WebPlotdigitizer. https://automeris.io/ WebPlotDigitizer [5/11/20].

\section{Discussion with Reviewers}

Piefrancesco Pagella: The authors only briefly discuss some studies concerning tRAS in human gingival and periodontal tissues. Are there reliable clinical studies concerning the association between tRAS and periodontitis? If yes, do their results go in the same direction of the animal studies discussed in this systematic review?

Authors: To the best of our knowledge, there are currently no clinical studies investigating the interaction between tRAS and periodontal diseases. We have recently started the first clinical study to assess and prove available preclinical evidence. The present study demonstrates that clinical trials are warranted to assess the impact of tRAS inhibition on periodontal diseases.

Editor's note: The Scientific Editor responsible for this paper was Thimios Mitsiadis. 